\title{
Long-term changes in coral assemblages under natural and anthropogenic stress in Jakarta Bay (1920-2005)
}

\author{
Sancia E.T. van der Meij ${ }^{\mathrm{a}, *}$, Suharsono $^{\mathrm{b}}$, Bert W. Hoeksema ${ }^{\mathrm{a}}$ \\ ${ }^{a}$ Department of Marine Zoology, Netherlands Centre for Biodiversity Naturalis, P.O. Box 9517, 2300 RA Leiden, The Netherlands \\ ${ }^{\mathrm{b}}$ Indonesian Institute of Sciences (LIPI), Research Centre for Oceanography, P.O. Box 4801, 11048 Jakarta, Indonesia
}

\section{A R T I C L E I N F O}

\section{Keywords:}

Biodiversity change

Global change

Kepulauan Seribu

Reef degradation

Scientific collections

Scleractinia

\begin{abstract}
A B S T R A C T
Coral reefs in Jakarta Bay have been subjected to scientific studies since the 1920s. Also from that time on biological collections were made. The reefs in the Jakarta Bay have been under long-term natural and anthropogenic stress. With the biological collections and historical documents the coral species richness in Jakarta Bay around 1920 was reconstructed. New data from this bay and the adjacent offshore Thousand Islands archipelago were obtained during a 2005 research expedition. A comparison of the coral assemblages between 1920 and 2005 reveals a clear decline in species numbers. The most prominent results include the near-shore disappearance of species belonging to the families Acroporidae, Milleporidae, and to a lesser extent Poritidae. The overall coral species composition of the reefs has changed considerably, which is partly reflected in a strong decline in coral species richness. About half the number of species recorded in 1920 was found again in 2005.
\end{abstract}

(c) 2010 Elsevier Ltd. All rights reserved.

\section{Introduction}

Species-rich coral reef ecosystems are under pressure worldwide from both natural and anthropogenic disturbance. Disturbances such as changes in seawater temperature and terrestrial run-off negatively influence coral reef ecosystems. The spatial complexity, biodiversity, and dynamic nature of these ecosystems are influenced by the intensity and variation in scale of both natural and anthropogenic disturbances (Wilkinson, 1996; Hoeksema, 2004; Fabricius, 2005; Wilson et al., 2006). Reefs are resilient to natural disturbances; but vulnerable to chronic stress, such as pollution and degradation of water quality (Wilkinson, 1993). Data on benthic marine ecosystems show directional changes in the structure of these ecosystems, which are related to the increasing occurrence, diversity, and scale of anthropogenic disturbances (Jackson et al., 2001; Hughes et al., 2003).

Throughout the tropics, centres of high reef coral diversity coincide with human population centres, and thus the greatest potential for species loss lies in these geographical areas (ChadwickFurman, 1996; Bryant et al., 1998; Burke et al., 2002). Coastal resources such as coral reefs are susceptible to considerable pressure from large metropolises. Coral reefs in areas with low human population densities are doing relatively well compared with reefs close to urban centres (Rogers, 1990; Roberts, 1993; Edinger et al., 1998; Rees et al., 1999). Regional anthropogenic impacts

\footnotetext{
* Corresponding author.

E-mail address: Sancia.vanderMeij@ncbnaturalis.nl (S.E.T. van der Meij).
}

are the largest threats to coral diversity and may interact with global climate change to exacerbate rates of local species extinctions (Chadwick-Furman, 1996).

Several studies have been published on the effects of anthropogenic pollution and sediments on local ecosystems over longer time periods. Based on historical data, McCulloch et al. (2003) found a five- to 10-fold increase in the delivery of sediments onto the inner Great Barrier Reef following the beginning of European settlement in the coastal area. Jackson et al. (2001) used historical research to show changes in coastal ecosystems, including work on coral reefs in the Caribbean and the Great Barrier Reef in Australia, showing dramatic phase shifts in dominant species on the reefs due to intensified human disturbances. A study in the Netherlands Antilles shows that rare coral species became extinct in permanent quadrats, and that other species became less abundant over a 20 year period, likely as a result of urbanisation (Bak and Nieuwland, 1995), whereas a study in the Red Sea shows a lack of recovery for disturbed reefs close to sources of pollution (Wielgus et al., 2003).

Despite the aforementioned studies on local changes in coral reef diversity, only little work has been carried out on multi-year changes in Indo-Pacific coral reef assemblages. In view of the importance of Indonesia's position in relation to the Coral Triangle (Hoeksema, 2007), there is a need for more long-term studies on reef coral diversity in this region. Furthermore, data from historical expeditions can provide information about distribution ranges of species and changes herein (van der Meij and Visser, in press). A 17-year study at Ko Phuket (Thailand) demonstrated the destructive effects of dredging and anomalously low sea water levels on 
coral communities (Brown et al., 2002). Research on mushroom coral assemblages near Singapore (1860s-2006) showed a loss of species richness, probably caused by anthropogenic disturbance (Hoeksema and Koh, 2009). Similar research carried out on the molluscan fauna of Jakarta Bay revealed a strong decline in species diversity over a 75 -year time period (van der Meij et al., 2009).

This study aims at documenting changes in coral reef biodiversity since the first half of the 20th century in Jakarta Bay and the adjacent Thousand Islands archipelago (Kepulauan Seribu), Java, Indonesia. This area has received increasing amounts of sewage since the early 1900s, and has been subjected to siltation, coastal development and coral mining. Furthermore, the corals have suf- fered from large-scale mortality resulting from natural stress, such as crown-of-thorns starfish (Acanthaster planci (Linnaeus, 1758)) outbreaks and bleaching resulting from ENSO (El Niño Southern Oscillation) events (Verstappen, 1988; Brown and Suharsono, 1990; Warwick et al., 1990; Hoeksema, 1991; Tomascik, 1997). Several reefs here have been extensively studied in the early 1900s, providing a baseline for the present study (e.g., Umbgrove, 1928a, b; Umbgrove and Verwey, 1929; Verwey, 1931; Delsman, 1937). Exploiting field data from an 85-year time span from a single area, combined with historical documents (notebooks, journals, and nautical maps) enables us to follow time trends in diversity on these coral reefs. The present study spans a period over which

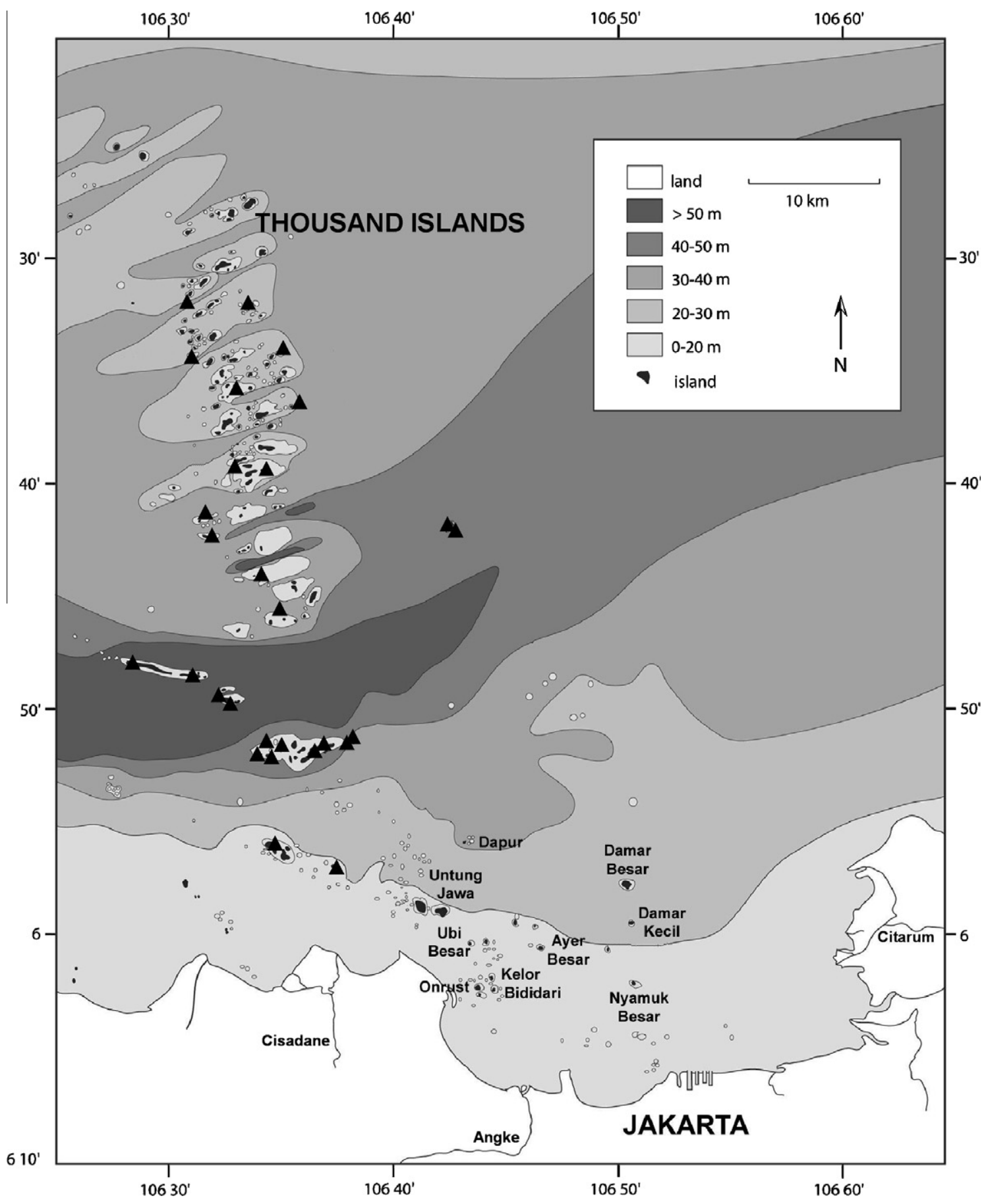

Fig. 1. Map of research area, with triangles indicating the 2005 sampling localities in the Thousand Islands archipelago. 
possible anthropogenic changes are documented, and offers the opportunity to detect changes in Jakarta Bay's coral fauna.

\section{Methods}

\subsection{Research area}

All research was carried out on reefs in Jakarta Bay and the adjacent Thousand Islands archipelago (Fig. 1). This reef complex extends from Jakarta's coastline to more than $80 \mathrm{~km}$ offshore in a NNW-SSE direction, perpendicular to the coast line, and $30 \mathrm{~km}$ from east to west $\left(5^{\circ} 47^{\prime}-5^{\circ} 24^{\prime} \mathrm{S}, 106^{\circ} 23^{\prime}-106^{\circ} 37^{\prime} \mathrm{E}\right)$. The reef complex consists of over 108 small ( $<10 \mathrm{ha}$ ) and low-lying ( $<3 \mathrm{~m}$ asl) coral cays (Brown, 1986). The Thousand Islands are separated from the bay area by a channel with a depth of about $100 \mathrm{~m}$ connected to the Sunda Strait (Umbgrove, 1929). Jakarta's extended agglomeration houses over 23 million inhabitants in an area of about 2700$\mathrm{km}^{2}$ and it is the world's largest conurbation located in close vicinity to a coral reef complex. Jakarta Bay is less than $30 \mathrm{~m}$ deep, with a bottom composed of terrigenous mud, coral sand, rubble, and coralline algae (Ongkosongo, 1989).

The bay is bordered by two capes, Tanjung Pasir to the west and Tanjung Karawang to the east. Three major rivers discharge fresh water and silt into Jakarta's reef area: the Cisadane, most westward and just outside the bay, the Angke, most southward inside the bay, and the Citarum, with a large river delta in the easternmost cape (Verstappen, 1953; Moll and Suharsono, 1986). The river Citarum carries large quantities of silt to the east side of the bay, confining the presence of coral reefs to its western part only (Umbgrove and Verwey, 1929). In addition, the bay receives highly polluted water from the rivers that drain metropolitan Jakarta. The present research area consists of a series of coral reefs ranging from those living in the polluted waters of Jakarta Bay to those in the clearer waters of the Java Sea (Brown, 1986).

\subsection{Water quality}

Secchi disk depth measurements near Onrust Island and Kelor Island did not show significant different results among 1929, 1985, and 1993 data, whereas for reefs further offshore a dramatic reduction in water transparency was noted (Tomascik et al., 1993). Little data is available on salinity and $\mathrm{Cl}$ content; in 1927 Kelor had salinity and $\mathrm{Cl}$ levels of respectively 29.4 and 17.8, Damar Kecil of 30.3 and 18.4, Nyamuk Besar of 31.2 and 18.9, and Bidadari of 31 and 18.8 (Umbgrove, 1928a). Historical data on the nutrient concentration $\left(\mathrm{PO}_{4}\right.$ and $\left.\mathrm{NO}_{3}\right)$ shows that Jakarta Bay has become progressively more eutrophic in time, paralleled by a strong reduction of the maximal depth of coral reef communities with stronger effects for islands further away (Tomascik et al., 1993). The effluents of the rivers Cisadane, Ciliwung, and Citarum have contributed greatly to this degradation of the bays' water quality. Studies in the western part of the bay have revealed that the degradation of water quality was due to household waste, whereas the studies in the eastern part of the bay re-

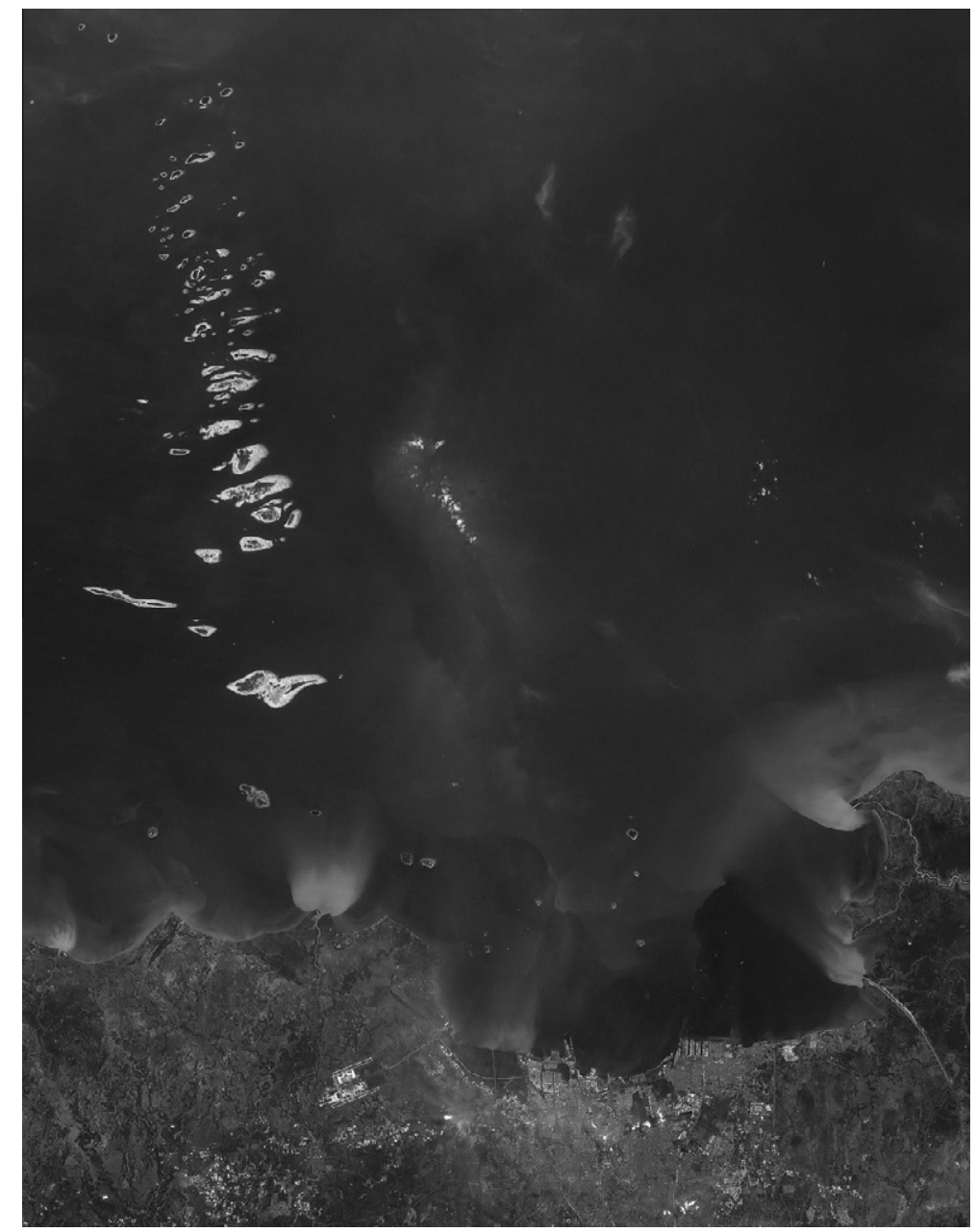

Fig. 2. Satellite photo showing river plumes (obtained via http://glcf.umiacs.umd.edu (Landsat), image taken in 2002). 
vealed that water pollution was caused by hydrocarbons and heavy metals. For $\mathrm{Pb}, \mathrm{Cd}, \mathrm{Hg}, \mathrm{Cu}$, and $\mathrm{Zn}$, the levels have reached or surpassed the permissible limits defined for standard water quality. Furthermore, the power plant at Tanjung Priok harbour produces vast amounts of cooling water, which results in thermal pollution (Sutamihardja, 1985).

\subsection{Data collection}

The scientific natural history collections in the Netherlands had much benefit from the availability of Jakarta's research facilities in the early 20th century: the Fisheries Station at Jakarta's fish market, which later became the Laboratory for the Investigation of the Sea, and some research facilities at Damar Besar Isl. Old collection labels indicate that maximum depths of $12 \mathrm{~m}$ were reached through dredging, helm diving, wading, and snorkelling. In the 1920s, mainly the exposed north-western sides of Jakarta Bay's reefs were sampled. The reefs Onrust, Damar Besar, and Ayer Besar were more thoroughly sampled in the early 1900s than other reefs in the bay, especially the reefs Kelor, Ubi Besar, and Bidadari seem to be undersampled. In addition to the collected material, historical documents were studied for further information on the reef condition in Jakarta Bay around 1920 and before.

In 2005,28 patch reefs throughout the Thousand Islands archipelago (including Jakarta Bay) were assessed. The reefs were sampled using the roving diver technique (Schmitt et al., 2002; Munro, 2005) in an environmental south-to-north, near-shore to off-shore gradient, with increasing distance from urbanization and river discharge (Figs 1 and 2). At the NW-side of each locality, one hour per observer was spent with the help of SCUBA equipment, reaching depths down to $30 \mathrm{~m}$, which was deeper than in 1920. Per encountered species, specimens were collected, photographed and/or noted on a PVC underwater writing board. Collected specimens were deposited in the Zoological Museum of Bogor (PPB-LIPI, presently at Cibinong). Due to a lack of trained observers, the genera Alveopora and Montipora were undersampled in 2005. After the

Table 1

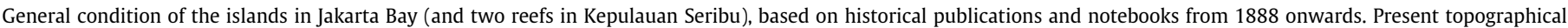
names (Indonesian Navy, 2002: map 86) are used, with the addition of alternative/old Dutch names (Dutch Navy, 1899: maps 86/87) in between brackets.

\begin{tabular}{|c|c|}
\hline Island/area & Remarks \\
\hline Jakarta Bay, Teluk Jakarta (Bay of Batavia) & $\begin{array}{l}\text { In comparison to the coasts of Singapore and Sumatra, the coast of NW Java around Jakarta is richer in species } \\
\text { (Sluiter, 1888). Hardenberg (1939) expresses the need for marine reserves to protect reefs from dredging and } \\
\text { other threats. Coral mining was common and carried out by means of explosives and crowbars, mainly } \\
\text { targeting Porites and Acropora. About } 12-15 \mathrm{dam}^{3} \text { of coral was landed annually, with exceptions of up to } \\
25 \mathrm{dam}^{3} \text {. The municipality of Jakarta was the largest buyer (Hardenberg, 1939). }\end{array}$ \\
\hline Ayer Besar (Hoorn) & $\begin{array}{l}\text { The coral island has been elevated with coral rubble for development, like building a restaurant (Hardenberg, } \\
\text { 1939). The reef was in good condition (Umbgrove, 1939). }\end{array}$ \\
\hline Ayer Kecil (= Nusi) (Haarlem) & $\begin{array}{l}\text { Young vegetation was planted on the cay. Large fields of Acropora were demolished in a storm (1934). } \\
\text { Hardenberg (1939) proposed a marine reserve as the reef is 'not inferior to the reefs of Ambon'. Coral mining } \\
\text { eventually caused this island to disappear (Stoddart, 1986; DeVantier et al., 1998). }\end{array}$ \\
\hline Ayer Sedang (= Gosong Putih) (Monnikendam) & $\begin{array}{l}\text { The cay was elevated with coral rubble (van Benthem-Jutting and Westenberg, unpublished; Hardenberg, } \\
\text { 1939). }\end{array}$ \\
\hline Bidadari (= Sakit) (Purmerend) & A leper colony was located on the island (van Benthem-Jutting and Westenberg, unpubl.). \\
\hline Cipir (= Burung) (Kuiper) & $\begin{array}{l}\text { Inhabited by staff of the meteorological observatory, which altered the flora (van Benthem-Jutting and } \\
\text { Westenberg, unpublished; Hardenberg, 1939). }\end{array}$ \\
\hline Damar Besar (Edam) & $\begin{array}{l}\text { Sluiter (1888) considered this reef, together with Nyamuk Kecil, to be most species-rich. The W and N side of } \\
\text { the reef consisted of live coral, whereas the E side consisted of dead coral (Boschma, unpublished, 1921-22; } \\
\text { Boschma, 1936). The island was elevated with coral rubble (Hardenberg, 1939). A house and lighthouse were } \\
\text { constructed, inhabited by the lighthouse keeper's family. }\end{array}$ \\
\hline Damar Kecil (=Monyet) (Alkmaar) & This island was privately owned (Verstappen, 1988). \\
\hline Kapal (Onrust) & $\begin{array}{l}\text { The documented history of this island goes back far. Before Dutch settlement (17th C.) the Banten kings used it } \\
\text { as a holiday resort. Since } 1610 \text { the Dutch East India Company (VOC) used the island as a trading post with } \\
\text { fortification, a naval base and pilgrim (haj) quarantine. In 1875-76 the Dutch Navy performed well drills, but } \\
\text { the water appeared undrinkable (Hooze, 1877; Sluiter, 1890). Settlements included a public aquarium. } \\
\text { Hardenberg (1939) mentioned that humans altered the island's flora. Currently a small human population lives } \\
\text { here (Attahiyyat, 2000). }\end{array}$ \\
\hline $\begin{array}{l}\text { Karang Karang Timbul (Bonte Ezel = Van Dorth?)/ } \\
\quad \text { Karang Cikasi (Broenda Droogte) }\end{array}$ & These reefs (first buoys outside of Tanjung Priok harbour) remained 2-3.5 m below water (Sluiter, 1888). \\
\hline Kelor (Kerkhof) & $\begin{array}{l}\text { Kelor has a history of coral mining and extensive fishing, as a result of which the reef is almost completely } \\
\text { destroyed (Verstappen, 1988). }\end{array}$ \\
\hline Nyamuk Besar (= Nirwana) (Leiden) & $\begin{array}{l}\text { From mangroves on the } \mathrm{N} \text { side of the island, a (possibly man-made) dam of dead coral stretched out into the } \\
\text { sea. On both sides of this dam a rich marine fauna could be found, most of which was unique for Jakarta Bay } \\
\text { (Sluiter, 1888). Montipora corals were abundant (Umbgrove, 1928b, 1939). Tourism, introduced in the 1960s, } \\
\text { was moved to Bidadari because of the rapid abrasion of the cay (Verstappen, 1988) Coral mining caused the } \\
\text { island's disappearance (Stoddart, 1986; DeVantier et al., 1998). }\end{array}$ \\
\hline Nyamuk Kecil (= Tala) (Enkhuizen) & $\begin{array}{l}\text { Nyamuk Kecil, together with Damar Besar, was considered to be the richest reef in Jakarta Bay (Sluiter, 1888) } \\
\text { The reef is largely dead because of water pollution and coral mining (Verstappen, 1988). }\end{array}$ \\
\hline Putri (Vader Smit) & $\begin{array}{l}\text { In } 1824 \text { Putri was a vegetated island (Verstappen, } 1988) \text {. Sluiter }(1888,1890) \text { mentioned that the reef partially } \\
\text { emerged during low tide, which he considered to be the first stage towards becoming a true coral cay. The } \\
\text { island degraded and became a shoal due to coral mining (Hardenberg, 1939). }\end{array}$ \\
\hline Ubi Besar (Rotterdam) & $\begin{array}{l}\text { Settlements of native fishermen altered the flora (van Benthem-Jutting and Westenberg, unpublished; } \\
\text { Hardenberg, 1939). Coral mining eventually caused the island to disappear (Stoddart, 1986; DeVantier et al., } \\
\text { 1998). }\end{array}$ \\
\hline Ubi Kecil (Schiedam) & $\begin{array}{l}\text { In } 1753 \text { Ubi Kecil was a wooded island (Verstappen, 1988), which lasted at least till 1904. The island became } \\
\text { eroded by the sea, and in } 1939 \text { the island was a bare sand cay (Hardenberg, 1939). Coral mining caused the } \\
\text { island's disappearance (Stoddart, 1986; DeVantier et al., 1998). }\end{array}$ \\
\hline $\begin{array}{l}\text { Jaga Utara (Noordwachter, northern part of } \\
\text { Kepulauan Seribu) }\end{array}$ & $\begin{array}{l}\text { According to Boschma (unpublished 1921-22), this island had a surf reef with large Pocillopora corals. The W } \\
\text { and N side of the reef were covered by living coral, whereas the E side consisted of dead coral. Various Fungia } \\
\text { species were observed in the } 1920 \text { s. Montipora corals were very abundant. }\end{array}$ \\
\hline $\begin{array}{l}\text { Menyawak/Rakittiang (= Kelapa) (Boompjeseiland, } \\
\text { Kepulauan Seribu) }\end{array}$ & $\begin{array}{l}\text { Although, the water around this reef contained less silt than around reefs in Jakarta Bay, the reef fauna here was } \\
\text { comparatively less rich (Wichmann, 1912; Zaneveld and Montagne, 1950). }\end{array}$ \\
\hline
\end{tabular}




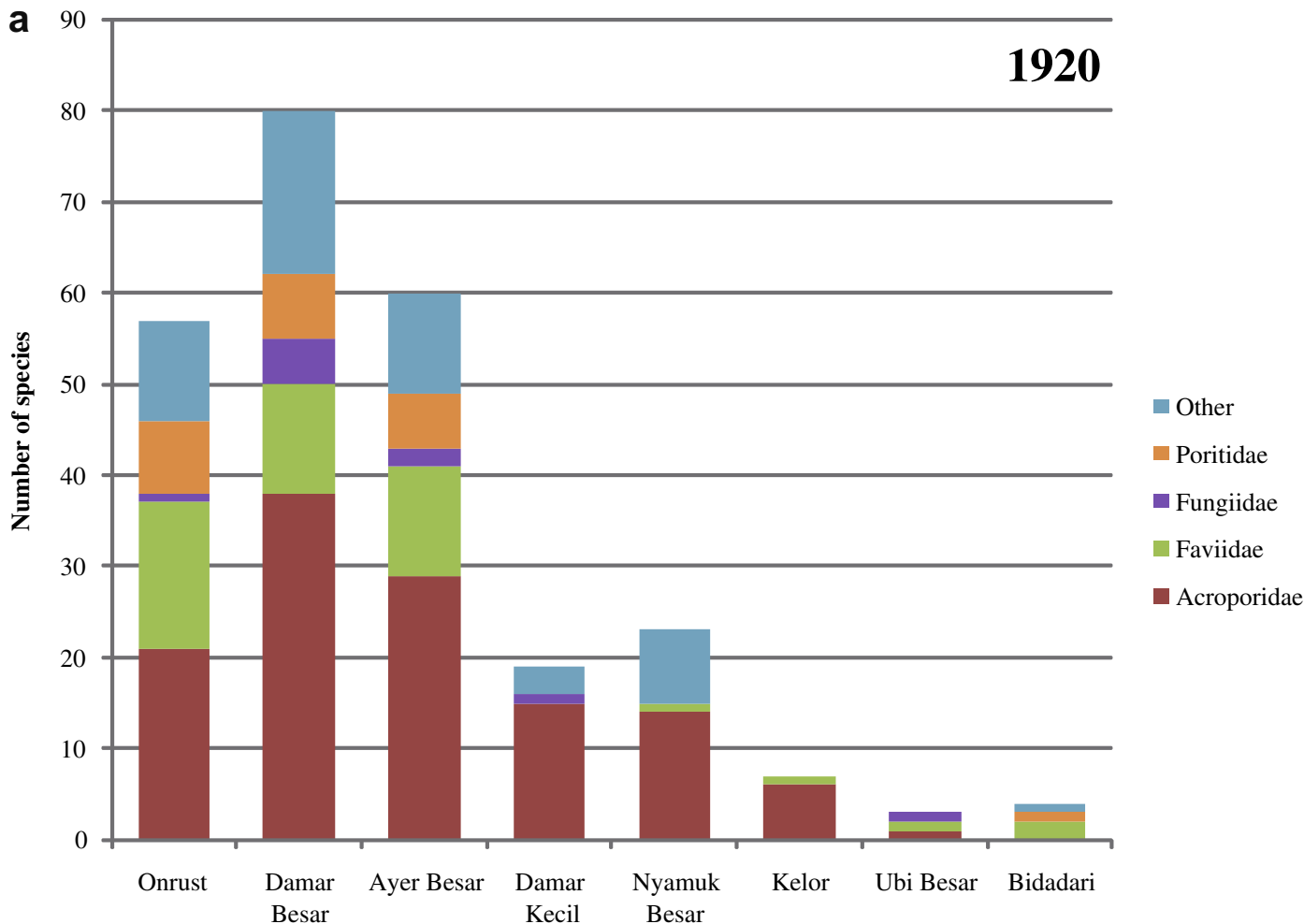

b

Reefs in Jakarta Bay

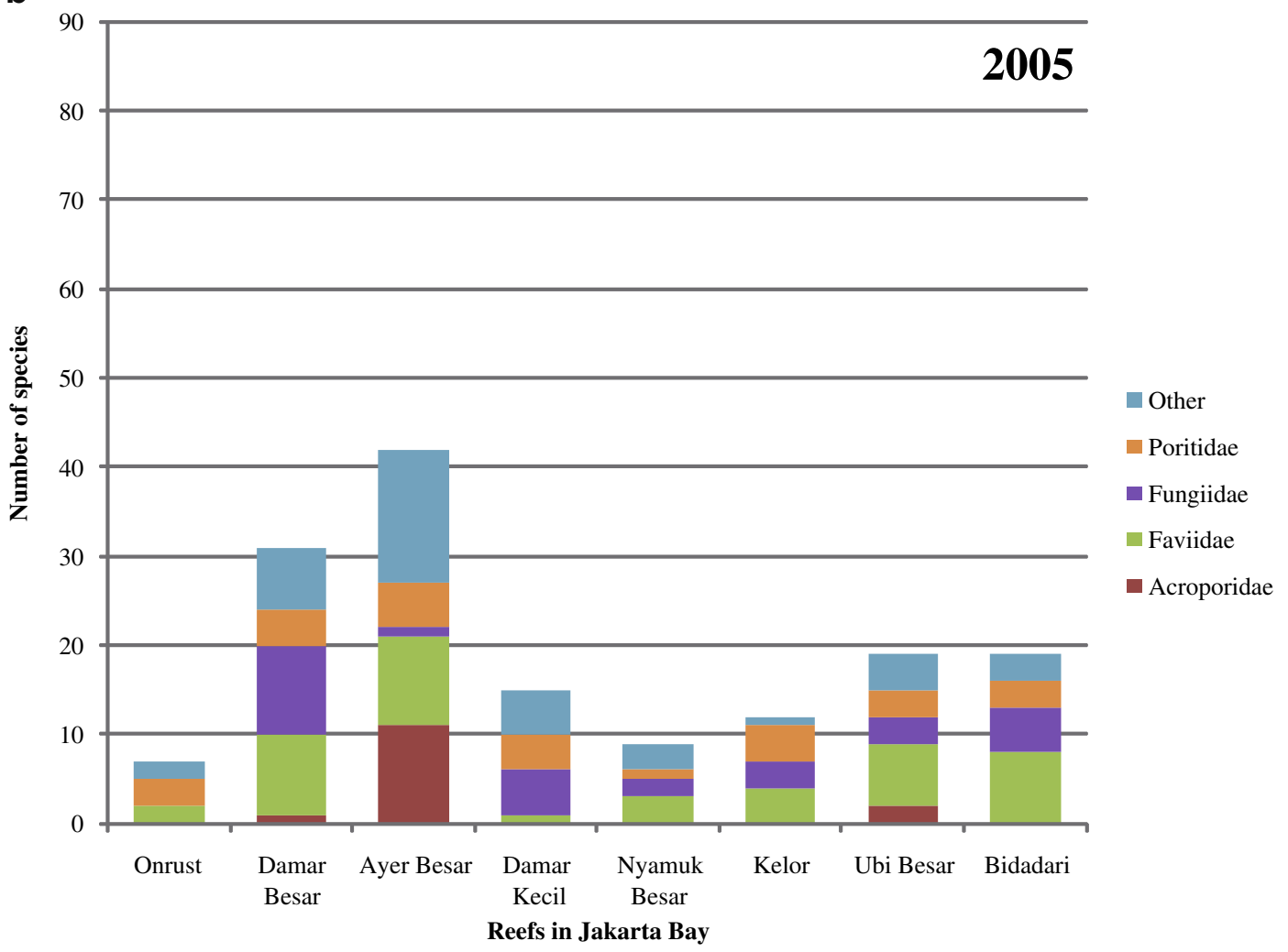

Fig. 3. ( $a$ and b) The number of coral species per reef in Jakarta Bay in 1920 and 2005, reefs are ranked from high to low impact in terms of species numbers.

2005 survey the historical coral collections of Naturalis were reidentified by the same researchers, to avoid observer bias.

Although additional data is available from surveys in 1985 and 1995 (Brown, 1986; Cleary et al., 2006, 2008), it was decided not to refer to those data in the present study. Different recording methods and observers, also between 1985 and 1995, and the lack of collections resulting from the 1985 and 1995 surveys, almost certainly influenced the data to the extent that its unreliability out- 
Table 2

List of coral species recorded from Jakarta Bay around 1920, based on literature and museum collections. All identifications have been updated to current knowledge (Wijsman-Best, 1972; Veron and Pichon, 1976; Veron et al., 1977; Veron and Pichon, 1980; Veron and Pichon, 1982; Veron and Wallace, 1984; Hoeksema, 1989; Wallace and Wolstenholme, 1998; Cairns et al., 1999; Wallace, 1999; Veron, 2000; Razak and Hoeksema, 2003). Species lists are compared with 2005 data from Jakarta Bay and Kepulauan Seribu. ${ }^{*}=$ records from Wallace and Wolstenholme (1998); ? = doubtful due to the absence of voucher specimens; $\mathrm{RMNH}=$ material present in NCB Naturalis, including the majority of specimens described by Umbgrove (1939).

\begin{tabular}{lllll}
\hline 1920 & 2005 & Reference \\
\cline { 3 - 3 } & & & \\
\cline { 3 - 4 } & Jakarta & Jakarta & Kep. & \\
Bay & Bay & Seribu & \\
& & & &
\end{tabular}

\section{Acroporidae $^{\mathrm{a}}$}

Acropora abrolhosensis Veron, $\quad \mathrm{x}$ 1985

A. abrotanoides (Lamarck, $\quad \mathrm{x}$ 1816)

A. aculeus (Dana, 1846)

A. acuminata (Verrill, 1864

A. anthocercis (Brook, 1893)

A. aspera (Dana, 1846)

A. austera (Dana, 1846)

A. carduus (Dana, 1846)

A. cerealis (Dana, 1846)

A. cytherea (Dana, 1846)

A. digitifera (Dana, 1846)

A. divaricata (Dana, 1846)

A. donei Veron and Wallace, 1984

A. echinata (Dana, 1846)

A. elseyi (Brook, 1892)

A. florida (Dana, 1846)

A. grandis (Brook, 1892)

A. hoeksemai Wallace, 1997

A. humilis (Dana, 1846)

A. hyacinthus (Dana, 1846)

A. indonesia Wallace, 1997

A. intermedia (Brook, 1891)

A. latistella (Brook, 1892)

A. longicyathus (M. Edwards \& Haime, 1860)

A. loripes (Brook, 1892)

A. lutkeni Crossland, 1952

A. microphthalma (Verrill, 1869)

A. millepora (Ehrenberg, 1834)

A. muricata (Linnaeus, 1758)

A. nana (Studer, 1878 )

A. nasuta (Dana, 1846)

A. pharaonis (M. Edwards \& Haime, 1860)

A. prolifera (Lamarck, 1816)

A. pulchra (Brook, 1891)

A. robusta (Dana, 1846 )

A. samoensis (Brook, 1891)

A. sarmentosa (Brook, 1892)

A. secale (Studer, 1878)

A. solitaryensis Veron and Wallace, 1984

A. spicifera (Dana, 1846)

A. subulata (Dana, 1846)

A. sukarnoi Wallace, 1997

A. tenuis (Dana, 1846)

A. tortuosa (Dana, 1846)

A. valida (Dana, 1846)

A. vaughani Wells, 1954

A. yongei Veron and Wallace, 1984

Astreopora myriophthalma $\quad \mathrm{x}$ (Lamarck, 1816)

Isopora brueggemanni (Brook, 1893)

Boschma, 1936

Hardenberg,

1939

RMNH

RMNH

Umbgrove and

Verwey, 1929

Boschma, 1936

RMNH

RMNH

RMNH

Umbgrove,

1928b

RMNH

RMNH

Boschma, 1936

RMNH

RMNH

RMNH

RMNH

Boschma, 1936

RMNH

RMNH

Umbgrove,

1939

RMNH
Table 2 (continued)

\begin{tabular}{lllll}
\hline 1920 & 2005 & & Reference \\
\cline { 3 - 4 } & & & & \\
\cline { 3 - 4 } & Jakarta & Jakarta & Kep. & \\
Bay & Bay & Seribu & \\
\hline
\end{tabular}

I. palifera (Lamarck, 1816)
Montipora aequituberculata
$\quad$ Bernard, 1897
M. angulata (Lamarck, 1816)
M. australiensis Bernard, 1897
M. cactus Bernard, 1897
M. calcarea Bernard, 1897
M. confusa Nemenzo, 1967
M. digitata (Dana, 1846)
M. effusa Dana, 1846
M. floweri Wells, 1954
M. foliosa (Pallas, 1766)

M. foveolata (Dana, 1846)

M. gaimardi Bernard, 1897

M. grisea Bernard, 1897

M. hispida (Dana, 1846)

M. hodgsoni Veron, 2000

M. hoffmeisteri Wells, 1954

M. informis (Bernard, 1897)

M. nodosa (Dana, 1846)

M. peltiformis Bernard, 1897

M. porites Veron, 2000

M. samarensis Nemenzo, 1967

M. spumosa (Lamarck, 1816)

M. stilosa (Ehrenberg, 1834)

M. turgescens Bernard, 1897

M. turtlensis Veron and

Wallace, 1984

M. undata Bernard, 1897

M. verrucosa (Lamarck, 1816)

Agariciidae

Coeloseris mayeri Vaughan, 1918

Pachyseris rugosa (Dana, 1846)

P. speciosa (Dana, 1846)

Pavona cactus (Forskål, 1775)

P. clavus (Dana, 1846)

P. decussata (Dana, 1846)

x $x \quad$ Boschma, 1936

$\mathrm{x} \quad \mathrm{RMNH}$

RMNH

RMNH

RMNH

Boschma, 1936

RMNH

RMNH

$\mathrm{RMNH}$

RMNH

Umbgrove,

1939

RMNH

RMNH

RMNH

RMNH

RMNH

RMNH

RMNH

RMNH

RMNH

RMNH

Hardenberg,

1939

RMNH

RMNH

RMNH

RMNH

Umbgrove,

1939

Umbgrove,

1939

Umbgrove,

1939

Umbgrove,

1939

Umbgrove,

1939

Umbgrove,

1939

Umbgrove,

1939

Umbgrove,

1939

Umbgrove,

1939

Umbgrove,

1939

Dendrophylliidae

Turbinaria peltata (Esper. 1794)

T. mesenterina (Lamarck, 1816)

T. reniformis Bernard, 1896

Euphylliidae

Euphyllia glabrescens

(Chamisso \& Eysenhardt, 1821)

Faviidae

Caulastrea furcata Dana, 1846

C. tumida Matthai, 1928

Cyphastrea chalcidicum (Forskål, 1775)

C. microphthalma (Lamarck 1816)

C. serailia (Forskål, 1775)
Umbgrove,

1939

RMNH

RMNH

Umbgrove and

RMNH

Umbgrove,

1939

Umbgrove,

1939

Umbgrove,

1939

Umbgrove,

1939
Verwey, 1929 
Table 2 (continued)

\begin{tabular}{|c|c|c|c|c|}
\hline & \multirow{2}{*}{$\begin{array}{l}1920 \\
\text { Jakarta } \\
\text { Bay }\end{array}$} & \multicolumn{2}{|l|}{2005} & \multirow{2}{*}{$\begin{array}{l}\text { Reference } \\
1920\end{array}$} \\
\hline & & $\begin{array}{l}\text { Jakarta } \\
\text { Bay }\end{array}$ & $\begin{array}{l}\text { Kep. } \\
\text { Seribu }\end{array}$ & \\
\hline $\begin{array}{l}\text { Diploastrea heliopora } \\
\quad \text { (Lamarck, 1816) }\end{array}$ & $\mathrm{x}$ & $\mathrm{x}$ & $\mathrm{x}$ & $\begin{array}{l}\text { Umbgrove, } \\
1939\end{array}$ \\
\hline $\begin{array}{l}\text { Echinopora gemmacea } \\
\quad(\text { Lamarck, 1816) }\end{array}$ & $\mathrm{x}$ & & $\mathrm{x}$ & $\begin{array}{l}\text { Umbgrove, } \\
1939\end{array}$ \\
\hline $\begin{array}{l}\text { E. hirsutissima (M. Edwards \& } \\
\text { Haime, 1849) }\end{array}$ & $\mathrm{x}$ & & $\mathrm{x}$ & RMNH \\
\hline E. horrida Dana, 1846 & $\mathrm{x}$ & & $\mathrm{x}$ & $\begin{array}{l}\text { Umbgrove, } \\
1939\end{array}$ \\
\hline E. lamellosa (Esper, 1795) & $\mathrm{x}$ & $\mathrm{x}$ & $\mathrm{x}$ & $\begin{array}{l}\text { Umbgrove, } \\
1939\end{array}$ \\
\hline Favia favus (Forskål, 1775) & $\mathrm{x}$ & $\mathrm{x}$ & $\mathrm{x}$ & Boschma, 1936 \\
\hline F. matthaii Vaughan, 1918 & & $\mathrm{x}$ & $\mathrm{x}$ & \\
\hline $\begin{array}{l}\text { F. maxima Veron, Pichon \& } \\
\text { Wijsman-Best, } 1977\end{array}$ & & $\mathrm{x}$ & & \\
\hline F. pallida (Dana, 1846) & $\mathrm{x}$ & $\mathrm{x}$ & $\mathrm{x}$ & $\begin{array}{l}\text { Umbgrove, } \\
1939\end{array}$ \\
\hline F. rotumana (Gardiner, 1899) & & $\mathrm{x}$ & $\mathrm{x}$ & \\
\hline F. speciosa (Dana, 1846) & $\mathrm{x}$ & $\mathrm{x}$ & $\mathrm{x}$ & $\begin{array}{l}\text { Umbgrove, } \\
1939\end{array}$ \\
\hline F. stelligera (Dana, 1846) & $\mathrm{x}$ & & $\mathrm{x}$ & $\begin{array}{l}\text { Umbgrove, } \\
1939\end{array}$ \\
\hline $\begin{array}{l}\text { Favites abdita (Ellis \& Solander, } \\
\text { 1786) }\end{array}$ & $\mathrm{x}$ & $\mathrm{x}$ & $\mathrm{x}$ & $\begin{array}{l}\text { Umbgrove, } \\
1939\end{array}$ \\
\hline F. acuticollis (Ortmann, 1889) & $\mathrm{x}$ & & & RMNH \\
\hline F. chinensis (Verrill, 1866) & $\mathrm{x}$ & $\mathrm{x}$ & $\mathrm{x}$ & RMNH \\
\hline $\begin{array}{l}\text { F. complanata (Ehrenberg, } \\
\text { 1834) }\end{array}$ & & $\mathrm{x}$ & $\mathrm{x}$ & \\
\hline $\begin{array}{l}\text { F. favosa (Ellis \& Solander, } \\
\qquad 1786)^{\mathrm{b}}\end{array}$ & $\mathrm{x}$ & & & $\begin{array}{l}\text { Umbgrove, } \\
1939\end{array}$ \\
\hline F. flexuosa (Dana, 1846) & $\mathrm{x}$ & & $\mathrm{x}$ & $\begin{array}{l}\text { Umbgrove, } \\
1939\end{array}$ \\
\hline F. halicora (Ehrenberg, 1834) & & $\mathrm{x}$ & $\mathrm{x}$ & \\
\hline F. pentagona (Esper, 1794) & $\mathrm{x}$ & $\mathrm{x}$ & $\mathrm{x}$ & $\begin{array}{l}\text { Umbgrove, } \\
1939\end{array}$ \\
\hline $\begin{array}{l}\text { Goniastrea aspera (Verrill, } \\
\text { 1865) }\end{array}$ & $\mathrm{x}$ & $\mathrm{x}$ & $\mathrm{x}$ & $\begin{array}{l}\text { Umbgrove, } \\
1939\end{array}$ \\
\hline G. favulus (Dana, 1846) & $\mathrm{x}$ & $\mathrm{x}$ & $\mathrm{x}$ & $\begin{array}{l}\text { Geologisch } \\
\text { Museum Artis }\end{array}$ \\
\hline G. pectinata (Ehrenberg, 1834) & $\mathrm{x}$ & & $\mathrm{x}$ & $\begin{array}{l}\text { Umbgrove, } \\
1939\end{array}$ \\
\hline G. retiformis (Lamarck, 1816) & $\mathrm{x}$ & $\mathrm{x}$ & $\mathrm{x}$ & $\begin{array}{l}\text { Umbgrove, } \\
1939\end{array}$ \\
\hline $\begin{array}{l}\text { Leptastrea pruinosa Crossland, } \\
\quad 1952\end{array}$ & & $\mathrm{x}$ & $\mathrm{x}$ & \\
\hline L. purpurea (Dana, 1846) & $\mathrm{x}$ & $\mathrm{x}$ & $\mathrm{x}$ & $\begin{array}{l}\text { Umbgrove, } \\
1939\end{array}$ \\
\hline L. transversa Klunzinger, 1879 & & $\mathrm{x}$ & $\mathrm{x}$ & \\
\hline $\begin{array}{l}\text { Leptoria phrygia (Ellis \& } \\
\quad \text { Solander, 1786) }\end{array}$ & $\mathrm{x}$ & & $\mathrm{x}$ & $\begin{array}{l}\text { Umbgrove, } \\
1939\end{array}$ \\
\hline Montastrea curta (Dana, 1846) & & $\mathrm{x}$ & $\mathrm{x}$ & \\
\hline $\begin{array}{l}\text { M. valenciennesii (M. Edwards } \\
\quad \text { \& Haime, 1848) }\end{array}$ & $\mathrm{x}$ & & & $\begin{array}{l}\text { Umbgrove, } \\
1939\end{array}$ \\
\hline $\begin{array}{l}\text { Oulastrea crispata (Lamarck, } \\
1816 \text { ) }\end{array}$ & $\mathrm{x}$ & $\mathrm{x}$ & $\mathrm{x}$ & $\begin{array}{l}\text { Umbgrove, } \\
1939\end{array}$ \\
\hline $\begin{array}{l}\text { Platygyra daedalea (Ellis \& } \\
\quad \text { Solander, 1786) }\end{array}$ & $\mathrm{x}$ & $\mathrm{x}$ & $\mathrm{x}$ & RMNH \\
\hline P. lamellina (Ehrenberg, 1834) & $\mathrm{x}$ & $\mathrm{x}$ & $\mathrm{x}$ & $\begin{array}{l}\text { Umbgrove, } \\
1939\end{array}$ \\
\hline P. pini Chevalier, 1971 & & $\mathrm{x}$ & $\mathrm{x}$ & \\
\hline $\begin{array}{l}\text { P. sinensis (M. Edwards \& } \\
\text { Haime, 1849) }\end{array}$ & $\mathrm{x}$ & & $\mathrm{x}$ & RMNH \\
\hline P. verweyi Wijsman-Best, 1976 & $\mathrm{x}$ & $\mathrm{x}$ & $\mathrm{x}$ & RMNH \\
\hline $\begin{array}{l}\text { P. yaeyamaensis Eguchi \& } \\
\text { Shirai, } 1977\end{array}$ & & $\mathrm{x}$ & & \\
\hline $\begin{array}{l}\text { Plesiastrea versipora (Lamarck, } \\
\text { 1816) }\end{array}$ & & $\mathrm{x}$ & $?$ & \\
\hline Fungiidae & & & & \\
\hline $\begin{array}{l}\text { Ctenactis albitentaculata } \\
\text { Hoeksema, } 1989\end{array}$ & & $\mathrm{x}$ & $\mathrm{x}$ & \\
\hline C. echinata (Pallas, 1766) & $\mathrm{x}$ & $\mathrm{x}$ & $\mathrm{x}$ & $\begin{array}{l}\text { Umbgrove, } \\
1939\end{array}$ \\
\hline Fungia concinna Verrill, 1864 & $x$ & $\mathrm{x}$ & $\mathrm{x}$ & Umbgrove, \\
\hline
\end{tabular}

Table 2 (continued)

\begin{tabular}{|c|c|c|c|c|}
\hline & \multirow{2}{*}{$\begin{array}{l}1920 \\
\text { Jakarta } \\
\text { Bay }\end{array}$} & \multicolumn{2}{|l|}{2005} & \multirow{2}{*}{$\begin{array}{l}\text { Reference } \\
1920\end{array}$} \\
\hline & & $\begin{array}{l}\text { Jakarta } \\
\text { Bay }\end{array}$ & $\begin{array}{l}\text { Kep. } \\
\text { Seribu }\end{array}$ & \\
\hline F. costulata Ortmann, 1889 & & $\mathrm{x}$ & $\mathrm{x}$ & \\
\hline F. fungites (Linnaeus, 1758) & $\mathrm{x}$ & $\mathrm{x}$ & $\mathrm{x}$ & $\begin{array}{l}\text { Umbgrove, } \\
1939\end{array}$ \\
\hline F. horrida Dana, 1846 & & $\mathrm{x}$ & $\mathrm{x}$ & \\
\hline $\begin{array}{l}\text { F. moluccensis Van der Horst, } \\
1919\end{array}$ & & $\mathrm{x}$ & $\mathrm{x}$ & \\
\hline $\begin{array}{l}\text { F. paumotensis Stutchbury, } \\
1833\end{array}$ & $\mathrm{x}$ & $\mathrm{x}$ & $\mathrm{x}$ & $\begin{array}{l}\text { Boschma 1921, } \\
1922\end{array}$ \\
\hline F. repanda Dana, 1846 & $\mathrm{x}$ & $\mathrm{x}$ & $\mathrm{x}$ & $\begin{array}{l}\text { Umbgrove, } \\
1939\end{array}$ \\
\hline F. scruposa Klunzinger, 1879 & $\mathrm{x}$ & $\mathrm{x}$ & $\mathrm{x}$ & $\begin{array}{l}\text { Umbgrove, } \\
1939\end{array}$ \\
\hline F. scutaria Lamarck, 1801 & $\mathrm{x}$ & & $\mathrm{x}$ & $\begin{array}{l}\text { Umbgrove, } \\
1939\end{array}$ \\
\hline $\begin{array}{l}\text { Heliofungia actiniformis (Quoy } \\
\text { \& Gaimard, 1833) }\end{array}$ & $\mathrm{x}$ & $\mathrm{x}$ & $\mathrm{x}$ & $\begin{array}{l}\text { Umbgrove, } \\
1939\end{array}$ \\
\hline Herpolitha limax (Esper, 1797) & $\mathrm{x}$ & $\mathrm{x}$ & $\mathrm{x}$ & $\begin{array}{l}\text { Umbgrove, } \\
1939\end{array}$ \\
\hline $\begin{array}{l}\text { Lithophyllon undulatum } \\
\text { Rehberg, } 1892\end{array}$ & & $\mathrm{x}$ & $\mathrm{x}$ & \\
\hline $\begin{array}{l}\text { Podabacia crustacea (Pallas, } \\
\text { 1766) }\end{array}$ & $\mathrm{x}$ & & $\mathrm{x}$ & $\begin{array}{l}\text { Boschma 1921, } \\
1922\end{array}$ \\
\hline $\begin{array}{l}\text { Polyphyllia talpina (Lamarck, } \\
\text { 1801) }\end{array}$ & $\mathrm{x}$ & $\mathrm{x}$ & $\mathrm{x}$ & $\begin{array}{l}\text { Umbgrove, } \\
1939\end{array}$ \\
\hline $\begin{array}{l}\text { Sandalolitha robusta (Quelch, } \\
\text { 1886) }\end{array}$ & $\mathrm{x}$ & $\mathrm{x}$ & $\mathrm{x}$ & $\begin{array}{l}\text { Umbgrove, } \\
1939\end{array}$ \\
\hline \multicolumn{5}{|l|}{ Helioporidae } \\
\hline $\begin{array}{l}\text { Heliopora coerulea (Pallas, } \\
\text { 1766) }\end{array}$ & $\mathrm{x}$ & $\mathrm{x}$ & $\mathrm{x}$ & $\begin{array}{l}\text { Umbgrove, } \\
1939\end{array}$ \\
\hline \multicolumn{5}{|l|}{ Merulinidae } \\
\hline $\begin{array}{l}\text { Hydnophora exesa (Pallas, } \\
\text { 1766) }\end{array}$ & $\mathrm{x}$ & $\mathrm{x}$ & $\mathrm{x}$ & $\begin{array}{l}\text { Umbgrove, } \\
1939\end{array}$ \\
\hline H. grandis Gardiner, 1906 & $\mathrm{x}$ & & & $\begin{array}{l}\text { Umbgrove, } \\
1939\end{array}$ \\
\hline H. microconos (Lamarck, 1816) & $\mathrm{x}$ & $\mathrm{x}$ & $\mathrm{x}$ & $\begin{array}{l}\text { Umbgrove, } \\
1939\end{array}$ \\
\hline H. rigida (Dana, 1846) & $\mathrm{x}$ & & $\mathrm{x}$ & $\begin{array}{l}\text { Umbgrove, } \\
1939\end{array}$ \\
\hline H. pilosa Veron, 1985 & & $\mathrm{x}$ & $\mathrm{x}$ & \\
\hline $\begin{array}{l}\text { Merulina ampliata (Ellis \& } \\
\quad \text { Solander, 1786) }\end{array}$ & $\mathrm{x}$ & & $\mathrm{x}$ & $\begin{array}{l}\text { Umbgrove, } \\
1939\end{array}$ \\
\hline $\begin{array}{l}\text { Scapophyllia cylindrica (M. } \\
\text { Edwards \& Haime, 1848) }\end{array}$ & $\mathrm{x}$ & & & $\begin{array}{l}\text { Umbgrove, } \\
1939\end{array}$ \\
\hline \multicolumn{5}{|l|}{ Milleporidae } \\
\hline $\begin{array}{l}\text { Millepora dichotoma Forskål, } \\
1775\end{array}$ & $\mathrm{x}$ & & $\mathrm{x}$ & $\begin{array}{l}\text { Umbgrove and } \\
\text { Verwey, } 1929\end{array}$ \\
\hline $\begin{array}{l}\text { M. platyphylla Hemprich \& } \\
\text { Ehrenberg, } 1834\end{array}$ & $\mathrm{x}$ & & & Boschma, 1936 \\
\hline M. intricata M. Edwards, 1857 & $\mathrm{x}$ & & $\mathrm{x}$ & RMNH \\
\hline M. latifolia Boschma, 1948 & $\mathrm{x}$ & & $\mathrm{x}$ & RMNH \\
\hline M. murrayi Quelch, 1884 & $\mathrm{x}$ & & & RMNH \\
\hline M. tenera Boschma, 1949 & $\mathrm{x}$ & \multicolumn{2}{|c|}{ Mussidae } & RMNH \\
\hline $\begin{array}{l}\text { Lobophyllia corymbosa } \\
\quad \text { (Forskål, 1775) }\end{array}$ & $\mathrm{x}$ & $\mathrm{x}$ & $\mathrm{x}$ & $\begin{array}{l}\text { Umbgrove, } \\
1939\end{array}$ \\
\hline L. costata (Dana, 1846) & $\mathrm{x}$ & & & $\begin{array}{l}\text { Umbgrove, } \\
1939\end{array}$ \\
\hline $\begin{array}{l}\text { L. hemprichii (Ehrenberg, } \\
\text { 1834) }\end{array}$ & $\mathrm{x}$ & & $\mathrm{x}$ & RMNH \\
\hline $\begin{array}{l}\text { Symphyllia agaricia M. } \\
\text { Edwards \& Haime, } 1849\end{array}$ & & $\mathrm{x}$ & $\mathrm{x}$ & \\
\hline $\begin{array}{l}\text { S. radians } \mathrm{M} \text {. Edwards \& } \\
\text { Haime, } 1849\end{array}$ & $\mathrm{x}$ & $\mathrm{x}$ & $\mathrm{x}$ & $\begin{array}{l}\text { Umbgrove, } \\
1939\end{array}$ \\
\hline S. recta (Dana, 1846) & $\mathrm{x}$ & $\mathrm{x}$ & $\mathrm{x}$ & $\begin{array}{l}\text { Umbgrove, } \\
1939\end{array}$ \\
\hline \multicolumn{5}{|l|}{ Oculinidae } \\
\hline $\begin{array}{l}\text { Galaxea astreata (Lamarck, } \\
\text { 1816) }\end{array}$ & $\mathrm{x}$ & & & $\begin{array}{l}\text { Umbgrove, } \\
1939\end{array}$ \\
\hline G. fascicularis (Linnaeus, 1767) & $\mathrm{x}$ & $\mathrm{x}$ & $\mathrm{x}$ & $\begin{array}{l}\text { Umbgrove, } \\
1939\end{array}$ \\
\hline $\begin{array}{l}\text { Simplastrea vesicularis } \\
\text { Umbgrove, } 1939\end{array}$ & $\mathrm{x}$ & & & $\begin{array}{l}\text { Umbgrove, } \\
1939\end{array}$ \\
\hline
\end{tabular}


Table 2 (continued)

\begin{tabular}{|c|c|c|c|c|}
\hline & \multirow{2}{*}{$\begin{array}{l}1920 \\
\text { Jakarta } \\
\text { Bay }\end{array}$} & \multicolumn{2}{|l|}{2005} & \multirow{2}{*}{$\begin{array}{l}\text { Reference } \\
1920\end{array}$} \\
\hline & & $\begin{array}{l}\text { Jakarta } \\
\text { Bay }\end{array}$ & $\begin{array}{l}\text { Kep. } \\
\text { Seribu }\end{array}$ & \\
\hline \multicolumn{5}{|l|}{ Pectiniidae } \\
\hline $\begin{array}{l}\text { Echinophyllia aspera (Ellis \& } \\
\quad \text { Solander, 1786) }\end{array}$ & $\mathrm{x}$ & $\mathrm{x}$ & $\mathrm{x}$ & $\begin{array}{l}\text { Umbgrove, } \\
1939\end{array}$ \\
\hline $\begin{array}{l}\text { Mycedium elephantotus (Pallas, } \\
1766 \text { ) }\end{array}$ & $\mathrm{x}$ & $\mathrm{x}$ & $\mathrm{x}$ & $\begin{array}{l}\text { Umbgrove, } \\
1939\end{array}$ \\
\hline Oxypora lacera (Verrill, 1864) & $\mathrm{x}$ & & & $\begin{array}{l}\text { Umbgrove, } \\
1939\end{array}$ \\
\hline Pectinia lactuca (Pallas, 1766) & $\mathrm{x}$ & & $\mathrm{x}$ & $\begin{array}{l}\text { Umbgrove, } \\
1939\end{array}$ \\
\hline P. paeonia (Dana, 1846) & $\mathrm{x}$ & & $\mathrm{x}$ & RMNH \\
\hline \multicolumn{5}{|l|}{ Pocilloporidae } \\
\hline $\begin{array}{l}\text { Pocillopora damicornis } \\
\quad(\text { Linnaeus, } 1758)^{\mathrm{c}}\end{array}$ & $\mathrm{x}$ & $\mathrm{x}$ & $\mathrm{x}$ & $\begin{array}{l}\text { Umbgrove, } \\
1939\end{array}$ \\
\hline $\begin{array}{l}\text { P. eydouxi M. Edwards \& } \\
\text { Haime, } 1860\end{array}$ & $\mathrm{x}$ & $\mathrm{x}$ & $\mathrm{x}$ & $\begin{array}{l}\text { Umbgrove, } \\
1939\end{array}$ \\
\hline $\begin{array}{l}\text { P. verrucosa (Ellis \& Solander, } \\
\text { 1786) }\end{array}$ & $\mathrm{x}$ & $?$ & $\mathrm{x}$ & $\begin{array}{l}\text { Umbgrove, } \\
1939\end{array}$ \\
\hline Seriatopora hystrix Dana, 1846 & $\mathrm{x}$ & $\mathrm{x}$ & $\mathrm{x}$ & $\begin{array}{l}\text { Umbgrove, } \\
1939\end{array}$ \\
\hline $\begin{array}{l}\text { Stylophora pistillata (Esper, } \\
\text { 1797) }\end{array}$ & $\mathrm{x}$ & $\mathrm{x}$ & $\mathrm{x}$ & $\begin{array}{l}\text { Umbgrove, } \\
1939\end{array}$ \\
\hline S. mordax (Dana, 1846) & $\mathrm{x}$ & & $\mathrm{x}$ & $\begin{array}{l}\text { Umbgrove, } \\
1939\end{array}$ \\
\hline \multicolumn{5}{|l|}{ Poritidae } \\
\hline $\begin{array}{l}\text { Alveopora verrilliana Dana, } \\
1872\end{array}$ & $\mathrm{x}$ & & & Boschma, 1936 \\
\hline $\begin{array}{l}\text { A. viridis (Quoy \& Gaimard, } \\
\text { 1833) }\end{array}$ & $\mathrm{x}$ & & & Boschma, 1936 \\
\hline Goniopora columna Dana, 1846 & $\mathrm{x}$ & $\mathrm{x}$ & $\mathrm{x}$ & $\begin{array}{l}\text { Umbgrove, } \\
1939\end{array}$ \\
\hline G. djiboutiensis Vaughan, 1907 & & $\mathrm{x}$ & $\mathrm{x}$ & \\
\hline $\begin{array}{l}\text { G. eclipsensis Veron and } \\
\text { Pichon, } 1982\end{array}$ & & $?$ & $\mathrm{x}$ & \\
\hline G. fructicosa Saville-Kent $1891^{\mathrm{d}}$ & $\mathrm{x}$ & $?$ & & RMNH \\
\hline $\begin{array}{l}\text { G. lobata M. Edwards \& Haime, } \\
1860\end{array}$ & & $\mathrm{x}$ & $\mathrm{x}$ & \\
\hline G. pendulus Veron, 1985 & & $?$ & & \\
\hline G. planulata (Ehrenberg, 1834) & & $\mathrm{x}$ & $\mathrm{x}$ & \\
\hline $\begin{array}{l}\text { G. stokesi M. Edwards \& } \\
\text { Haime, } 1851\end{array}$ & & $\mathrm{x}$ & $\mathrm{x}$ & \\
\hline G. stutchburyi Wells, 1955 & & $\mathrm{x}$ & $\mathrm{x}$ & \\
\hline G. tenuidens Quelch, 1886 & $\mathrm{x}$ & $\mathrm{x}$ & $\mathrm{x}$ & $\begin{array}{l}\text { Umbgrove, } \\
1939\end{array}$ \\
\hline Porites annae Crossland, 1952 & $\mathrm{x}$ & $?$ & & RMNH \\
\hline P. aranetai Nemenzo, 1955 & $\mathrm{x}$ & & $\mathrm{x}$ & RMNH \\
\hline P. attenuata Nemenzo, 1955 & $\mathrm{x}$ & & & RMNH \\
\hline P. australiensis Vaughan, 1918 & $\mathrm{x}$ & $\mathrm{x}$ & $\mathrm{x}$ & RMNH \\
\hline P. compressa Dana, 1846 & $\mathrm{x}$ & & & $\begin{array}{l}\text { Umbgrove, } \\
1939\end{array}$ \\
\hline P. cylindrica Dana, 1846 & $\mathrm{x}$ & $\mathrm{x}$ & $\mathrm{x}$ & RMNH \\
\hline P. deformis Nemenzo, 1955 & $\mathrm{x}$ & & $\mathrm{x}$ & RMNH \\
\hline P. lichen Dana, 1846 & $\mathrm{x}$ & $\mathrm{x}$ & $\mathrm{x}$ & RMNH \\
\hline P. lobata Dana, 1846 & $\mathrm{x}$ & $\mathrm{x}$ & $\mathrm{x}$ & $\begin{array}{l}\text { Umbgrove, } \\
1939\end{array}$ \\
\hline $\begin{array}{l}\text { P. lutea M. Edwards \& Haime, } \\
1860\end{array}$ & $\mathrm{x}$ & $\mathrm{x}$ & $\mathrm{x}$ & $\begin{array}{l}\text { Umbgrove, } \\
1939\end{array}$ \\
\hline P. murrayensis Vaughan, 1918 & & $\mathrm{x}$ & & \\
\hline P. nigrescens Dana, $1846^{\mathrm{e}}$ & $\mathrm{x}$ & $\mathrm{x}$ & $\mathrm{x}$ & RMNH \\
\hline P. pukoensis Vaughan, $1907^{\mathrm{f}}$ & $\mathrm{x}$ & & & RMNH \\
\hline P. rus (Forskål, 1775) & $\mathrm{x}$ & & $\mathrm{x}$ & $\begin{array}{l}\text { Umbgrove, } \\
1939\end{array}$ \\
\hline P. solida (Forskål, 1775) & $\mathrm{x}$ & $\mathrm{x}$ & $\mathrm{x}$ & RMNH \\
\hline P. stephensoni Crossland, 1952 & $\mathrm{x}$ & $\mathrm{x}$ & $\mathrm{x}$ & $\begin{array}{l}\text { Umbgrove, } \\
1939\end{array}$ \\
\hline $\begin{array}{l}\text { P. vaughani Crossland, } 1952 \\
\text { Siderastreidae }\end{array}$ & $\mathrm{x}$ & $?$ & $\mathrm{x}$ & RMNH \\
\hline $\begin{array}{l}\text { Psammocora contigua (Esper, } \\
\text { 1797) }\end{array}$ & $\mathrm{x}$ & $\mathrm{x}$ & $\mathrm{x}$ & $\begin{array}{l}\text { Umbgrove, } \\
1939\end{array}$ \\
\hline $\begin{array}{l}\text { P. digitata M. Edwards \& } \\
\text { Haime, } 1851\end{array}$ & $\mathrm{x}$ & & $\mathrm{x}$ & RMNH \\
\hline P. folium Umbgrove, 1939 & $\mathrm{x}$ & & & $\begin{array}{l}\text { Umbgrove, } \\
1939\end{array}$ \\
\hline
\end{tabular}

Table 2 (continued)

\begin{tabular}{|c|c|c|c|c|}
\hline & \multirow{2}{*}{$\begin{array}{l}1920 \\
\text { Jakarta } \\
\text { Bay }\end{array}$} & \multicolumn{2}{|l|}{2005} & \multirow{2}{*}{$\begin{array}{l}\text { Reference } \\
1920\end{array}$} \\
\hline & & $\begin{array}{l}\text { Jakarta } \\
\text { Bay }\end{array}$ & $\begin{array}{l}\text { Kep. } \\
\text { Seribu }\end{array}$ & \\
\hline $\begin{array}{l}\text { P. haimeana M. Edwards \& } \\
\text { Haime, } 1851\end{array}$ & $\mathrm{x}$ & $\mathrm{x}$ & $\mathrm{x}$ & $\begin{array}{l}\text { Umbgrove, } \\
1939\end{array}$ \\
\hline $\begin{array}{l}\text { P. profundacella Gardiner, } \\
1898\end{array}$ & & $\mathrm{x}$ & $\mathrm{x}$ & \\
\hline P. superficialis Gardinger, 1898 & & $\mathrm{x}$ & $\mathrm{x}$ & \\
\hline $\begin{array}{l}\text { Pseudosiderastrea tayami Yabe } \\
\text { \& Sugiyama, } 1935 \\
\text { Tubiporidae }\end{array}$ & & $\mathrm{x}$ & $\mathrm{x}$ & \\
\hline $\begin{array}{l}\text { Tubipora musica (Linnaeus, } \\
\text { 1758) }\end{array}$ & $\mathrm{x}$ & & $\mathrm{x}$ & $\begin{array}{l}\text { Boschma 1921, } \\
1922\end{array}$ \\
\hline
\end{tabular}

a Verwey informed Umbgrove that he intended to publish a monograph on the Acroporidae, including the description of 21 new species from Jakarta Bay. This unpublished manuscript was available to Wallace (1999).

b Favites favosa is a possible synonym of F. abdita, F. chinensis and F. flexuosa (Veron et al., 1977; Scheer,1990). Its identity is unclear, since Umbgrove (1939) did not indicate any types.

c Boschma (1936) refers to Pocillopora clavicornis (Esper). This is a most likely a nomen nudum and perhaps an unintended mix-up of $P$. clavaria Ehrenberg, 1834 and P. damicornis (Linnaeus, 1758), since it was not mentioned by Esper (Scheer, 1990).

d Recorded as Goniopora arbuscula spec. nov. by Umbgrove, 1939 (a junior synonym).

e Hardenberg (1939) mentioned Porites mucronata Dana, 1846, which was originally published as a variety of $P$. nigrescens.

${ }^{f}$ New record, formerly only known from Hawaii (Veron, 2000).

weighs the need for more. Especially recently revised coral taxa, such as the Fungiidae (Hoeksema, 1989) and Acropora (Wallace, 1999) show notable differences in observed species between years.

Data over an 85-year time period is compared and therefore differences in collection methods are unavoidable. Rigorous statistical analyses cannot be conducted, but the value of this historical dataset is compelling in a temporal sense. A total of 16 families of corals (including mostly zooxanthellate species of Scleractinia, as well as Helioporidae, Milleporidae, and Tubiporidae) are included in this study.

\section{Results}

\subsection{Historical data}

Information derived from historical data was used to reconstruct the environment of Jakarta Bay in the late 1800s and early 1900s. Already in 1927, anthropogenic influences were measured at Onrust Reef, and Zaneveld and Verstappen (1952) and Verstappen (1953) proposed to exclude it from further surveys. All derived information is summarized per reef (Table 1 ).

Sluiter (1888) discussed the possible formation of two shallow patch reefs close to Tanjung Priok harbour; Karang Prigem (St. Nicolaas Droogte) and Karang Cikasi (Broenda Droogte). Furthermore, he described the development of a new reef between Nyamuk Kecil and Nyamuk Besar, which was not indicated on any map (Dutch Navy, 1899). On several maps (see e.g., Umbgrove, 1939) islands and sandbanks are shown for which no or limited further information exists, such as Neptunus Droogte, Neerstuk, and Wapen van Purmerend.

\subsection{Comparison 1920-2005}

In the 1920s, the reefs Onrust, Damar Besar and Ayer Besar were studied most intensively, with only limited use of diving techniques, which were less advanced at that time. All three is- 
Table 3

Coral species observed in the offshore Thousand Islands archipelago in 2005, but not encountered in Jakarta Bay in 1920 and 2005

\author{
Acroporidae \\ Acropora clathrata (Brook, 1891) \\ A. horrida (Dana, 1846) \\ A. microclados (Ehrenberg, 1834) \\ A. selago (Studer, 1878) \\ A. spathulata (Brook, 1891) \\ A. striata (Verrill, 1866) \\ Montipora altasepta Nemenzo, 1967 \\ M. hirsuta Nemenzo, 1967 \\ M. mollis Bernard, 1897 \\ Agariciidae \\ Gardineroseris planulata (Dana, 1846) \\ Leptoseris amitoriensis Veron, 1990 \\ L. explanata Yabe \& Sugiyama, 1941 \\ L. foliosa Dinesen, 1980 \\ L. hawaiiensis Vaughan, 1907 \\ L. mycetoseroides Wells, 1954 \\ L. papyracea (Dana, 1846) \\ L. scabra Vaughan, 1907 \\ L. tubulifera Vaughan, 1907 \\ Pavona explanulata (Lamarck, 1816) \\ P. maldivensis (Gardiner, 1905) \\ P. minuta Wells, 1954 \\ Dendrophylliidae \\ Tubastrea coccinea Lesson, 1829 \\ T. micranthus (Ehrenberg, 1834) \\ Euphylliidae \\ Euphyllia cristata Chevalier, 1971 \\ Physogyra lichtensteini (M. Edwards \& Haime, 1851) \\ Plerogyra sinuosa (Dana, 1846) \\ Faviidae \\ Caulastrea echinata (M. Edwards \& Haime, 1849) \\ Echinopora mammiformis (Nemenzo, 1959) \\ E. pacificus Veron, 1990 \\ Favia helianthoides Wells, 1954 \\ F. maritima (Nemenzo, 1971) \\ F. rosaria Veron, 2000 \\ F. rotundata (Veron, Pichon \& Wijsman-Best, 1977) \\ F. veroni Moll \& Best, 1984 \\ Favites paraflexuosa Veron, 2000 \\ Goniastrea edwardsi Chevalier, 1971 \\ G. minuta Veron, 2000 \\ G. palauenis (Yabe, Sugiyama \& Eguchi, 1936) \\ Monstastrea magnistellata Chevalier, 1971 \\ Oulophyllia bennettae (Veron, Pichon \& Best, 1977) \\ O. crispa (Lamarck, 1816) \\ Fungiidae \\ Ctenactis crassa (Dana, 1846) \\ Fungia cyclolites Lamarck, 1816 \\ F. fragilis (Alcock, 1893) \\ F. granulosa Klunzinger, 1879 \\ F. sinensis (M. Edwards \& Haime, 1851) \\ F. spec. nov. \\ F. tenuis Dana, 1846 \\ F. vaughani Boschma, 1923 \\ Halomitra pileus (Linnaeus, 1758) \\ Lithophyllon mokai Hoeksema, 1989 \\ Sandolitha dentata Quelch, 1884 \\ Mussidae \\ Acanthastrea echinata (Dana, 1846) \\ Lobophyllia hataii Yabe, Sugiyama \& Eguchi, 1936 \\ Symphyllia valenciennesi M. Edwards \& Haime, 1849 \\ Pectiniidae \\ Echinophyllia echinata (Saville-Kent, 1871) \\ E. patula (Hodgson \& Ross, 1981) \\ Oxypora glabra Nemenzo, 1959 \\ Pectinia alcicornis (Saville-Kent, 1871) \\ Poritidae \\ Alveopora cf. catalai Wells, 1968 \\ Goniopora burgosi Nemenzo, 1955 \\ G. cf. cellulosa Veron, 1990 \\ G. minor Crossland, 1952 \\ G. cf. norfolkensis Veron and Pichon, 1982 \\ G. palmensis Veron and Pichon, 1982 \\ G. polyformis Zou, 1980
}

G. somaliensis Vaughan, 1907

Porites cumulatus Nemenzo, 1955

P. cf. mayeri Vaughan, 1918

P. monticulosa Dana, 1846

Siderastreidae

Coscinaraea exaesa (Dana, 1846)

C. wellsi Veron and Pichon, 1980

Psammocora nierstraszi Van der Horst, 1921

P. obtusangula (Lamarck, 1816)

lands show a dramatic decline in species richness between 1920 and 2005 (Fig. 3a and b). Some genera surveyed in the 1920s were not thoroughly sampled in the 2005 campaign (Alveopora, Montipora), or have been synonymised since (Tridacophyllia). Based on our data, the total number of stony coral species in the Jakarta Bay sums up to 190 in 1920, whereas in 2005105 species were recorded for Jakarta Bay and 160 for the more offshore Thousand Islands (Table 2), and 243 for the whole area, consisting of 236 zooxanthellate scleractinians, two azooxanthellate scleractinians, three milleporids and two octocorals (Tables 2 and 3). The total number of stony corals recorded over the period 1920-2005 is 296 for the whole area (Jakarta Bay and Thousand Islands), 286 of which are zooxanthellate scleractinians (Tables 2 and 3 ).

Umbgrove (1939) described Simplastrea vesicularis as a new coral species with Onrust Reef as type locality, but this species has not been found since in Jakarta Bay. In 1920, 57 coral species were encountered at Onrust Reef (Fig. 3a). Djohani (1994) recorded five species for Onrust (Leptastrea pruinosa, Oulastrea crispata, Platygyra pini, Porites lutea, and Turbinaria peltata), and in 2005 seven species were found (Oulastrea crispata, Pavona decussata, Platygyra pini, Porites lichen, P. murrayensis, P. solida, and Psammocora digitata). The reef flat has completely disappeared and only small colonies of the encountered species were found. According to Tomascik et al. (1993), Umbgrove (1939) recorded 96 species for Nyamuk Besar Island, 16 of which remained in 1993. However, Umbgrove did not record species per island, but for the entire Jakarta Bay.

Species with the most striking changes in richness belong to the coral families Acroporidae and Milleporidae. The species composition of the reefs in Jakarta Bay has changed considerably. In 1920, the Acroporidae were important contributors to Jakarta Bay's species numbers, but they became heavily reduced since then (Figs. 3 and 4). Some smaller families were also strongly affected. The Milleporidae (fire corals) appear to have disappeared from the bay, where they used to be common (Fig. 4, Table 2; Razak and Hoeksema, 2003). The increase in the encountered number of Fungiidae (Figs. 3 and 4) is an artefact of taxonomic resolution and observer bias, in that Hoeksema, who is a coral taxonomist specialized in fungiids, participated in the 2005 survey. Hoeksema also collected the data on the Siderastreidae.

\subsection{Thousand Islands 2005}

Some species that were not observed in Jakarta Bay in 2005 (but present in 1920), could still be found in the more offshore Thousand Islands archipelago. This is particularly true for species from strongly affected families such as Acroporidae and Milleporidae. The number of Acropora species (46) represented in Jakarta Bay in 1920 was even $10 \%$ more than the number (41) recorded in the Thousand Islands in 2005 (Tables 2 and 3). The Agariciidae, Faviidae, and Fungiidae appear to be much better represented on the offshore reefs of the Thousand Islands archipelago than they have ever been in Jakarta Bay (Tables 2 and 3). 


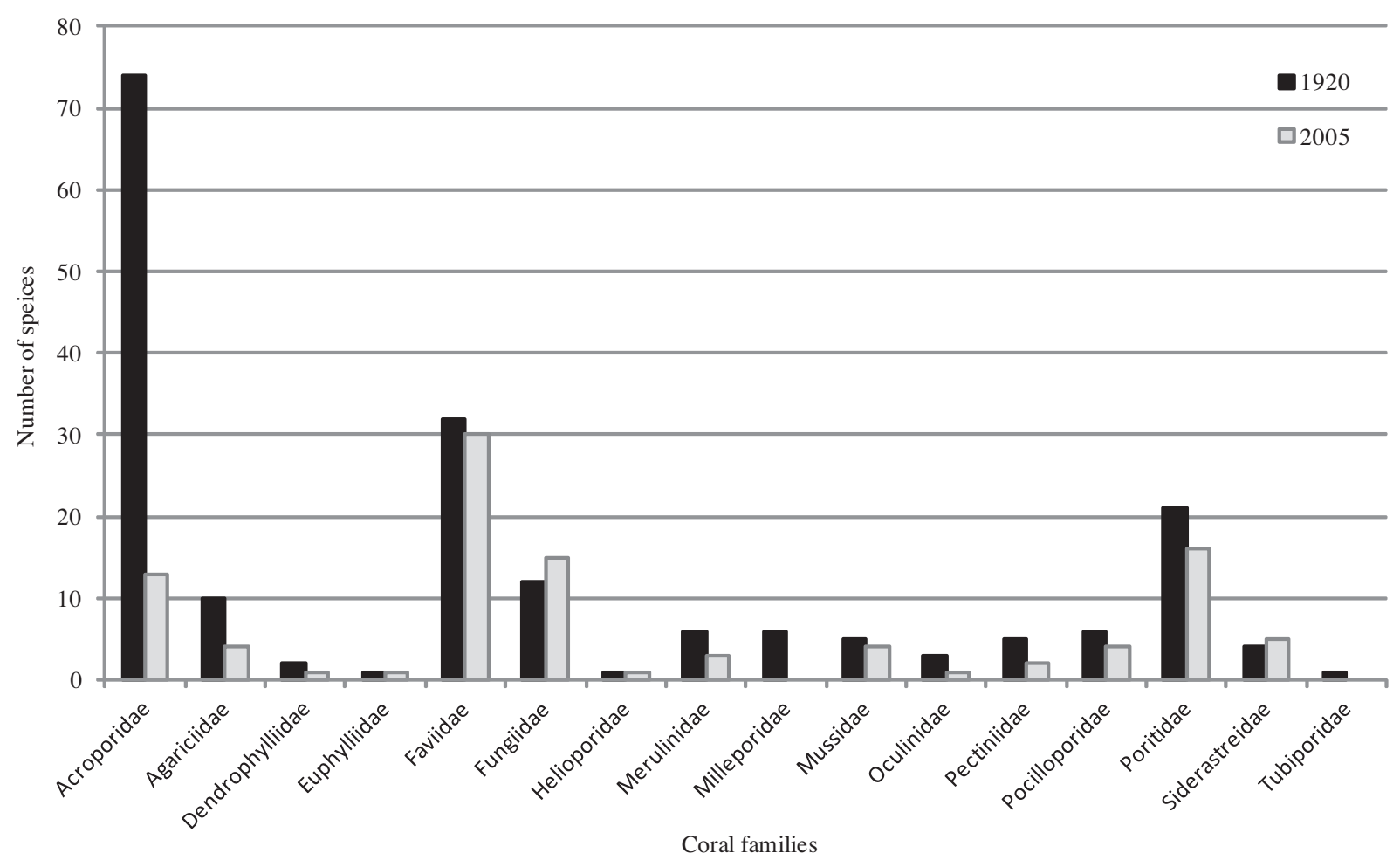

Fig. 4. Number of coral species, per family, found in Jakarta Bay in 1920 and 2005.

\section{Discussion}

There is no doubt that the demise of the Jakarta Bay coral reef fauna has been the price of development, since in the past it had some of the most diverse coral reefs in the Java Sea (Sluiter, 1888; Umbgrove, 1939; Tomascik et al., 1997). Nevertheless, already in the 1920 s the reefs were reported to be influenced by human impact (Zaneveld and Verstappen, 1952; Verstappen, 1953) and their degradation still continues. Jakarta's conurbation as a major source for pollution in the coastal zone and on nearby islands is clearly demonstrated by studies on beach litter, which indicate that most garbage on reefs consists of domestic and industrial refuse from Jakarta (Willoughby, 1986). The proximity of Jakarta also has secondary effects on pollution, since tourists from Jakarta and increased ship traffic to and from its harbour also cause pollution on the reefs (Uneputty and Evans, 1997; Willoughby et al., 1997).

This multi-decade study shows the increased human impact from Jakarta's coast and hinterland on the reefs in Jakarta Bay and the adjacent archipelago. With the use of historical scientific collections baselines can be found for human-induced impact on the degradation of coral reefs close to major human settlements. While different collectors and collecting methods have influenced the data presented in this article, the observed decline in species numbers is indisputable. The present study shows the result of prolonged stress on the species composition of reefs in Jakarta Bay, in this case reflected by the loss of species belonging to Acroporidae, Milleporidae, and to a lesser extent Poritidae. The strong decline of Acroporidae and Milleporidae is most likely related to their higher susceptibility to stress, as Marshall and Baird (2000) observed at the Great Barrier Reef. Research in the Red Sea also shows a reduction in abundance of the hydrocoral Millepora (Wielgus et al., 2003). According to Edinger and Risk (2000) Acropora, tabular non-Acropora, Heliopora, and Millepora are disturbanceadapted ruderals, whereas (sub-)massive corals are more stresstolerant. This agrees with the present data to a certain extent, but sampling methods and observer bias are also likely important factors in the observed differences.

The present results complement recent work on the molluscan fauna in Jakarta Bay (van der Meij et al., 2009). The decline in coral species richness of Jakarta Bay as compared to the higher species richness of the Kepulauan Seribu resembles the changes reported for the molluscan fauna. Damar Besar and Nyamuk Besar Isl. are strongly affected reefs, and show a similar decline in mollusc species richness. At these reefs no conid, cypraeid or nassariid snails, nor venerid bivalves, were observed in 2005. The coral fauna in Jakarta Bay in 2005 consisted of about $55 \%(n=190)$ of the number of species recorded in 1920. For molluscs the difference is larger, only about $33 \%(n=171)$ of the number of species recorded in $1937 / 38$ was still present in 2005. The depauperation of the reef coral fauna likely influenced the presence of reef-dwelling molluscs. The combined results suggest that other coral reef species, depending on corals for food, substrate and shelter, may also have become affected.

The major human-induced stressors on coral reefs have been identified for decades, including sewage, siltation, industrial discharge, urban development, and destructive fishing methods (Risk, 1999). A high load of suspended particulate matter may cause sediment accumulation on coral tissues and thereby stress and reduced growth and eventual smothering and death (Brown and Howard, 1985; Rogers, 1990). At regional scales, it is often difficult to assess causal relationships between increasing terrigenous runoff and reef degradation. Pollution effects and other disturbances are typically confounded, since historical data are often missing and reef communities change naturally along gradients from oceanic conditions to coastal conditions (Fabricius, 2005). Differentiation between natural and human impacts on coral reefs is hardly possible, since very few reef ecosystems are free of anthropogenic influence (Wilkinson, 1993). Hughes and Connell (1999) argue that human impact should be viewed in the context of the natural dynamics of coral reefs. Based on case studies in Jamaica and Australia, they show that recovery of reefs affected by natural factors 
(e.g., hurricanes, extremely low tides, predator outbreaks, ENSOevents) is rapid compared to reefs affected by predominantly anthropogenic impact. Coral assemblages suffering from chronic (usually anthropogenic) impact recovered in only $27 \%$ of cases, compared to $69 \%$ for acute impacts (Connell, 1997). Apart from local anthropogenic stress, the coral reefs in the Jakarta Bay and Thousand Islands archipelago have also endured large-scale mortality resulting from natural stress (Brown and Suharsono, 1990; Hoeksema, 1991; Tomascik et al., 1997). The mass coral mortality in 1983 resulting from bleaching may not be relevant to the present results, since Acropora species in the Thousand Islands already showed signs of recovery within five years (Brown and Suharsono, 1990). Whether the coral fauna in Jakarta Bay became permanently affected by bleaching or has recovered afterwards, remains uncertain.

When considering the importance of coral reefs for the livelihood of millions of people in developing countries (e.g., fishing, tourism, and coastal protection) the need for coral reef conservation in the vicinity of large cities is obvious. Sunier (1923) considered the coral reefs in Jakarta Bay an extremely rich field for scientific studies. Nowadays this still holds, but for other reasons. The vast anthropogenic impacts on Jakarta Bay make it a perfect location to study human impact on coastal ecosystems. Current social and economic developments in densely populated regions include accelerated urbanization of coastal margins, increasing the pressure on these species-rich ecosystems (Bryant et al., 1998; Burke et al., 2002). Regarding the predicted population growth in Southeast Asia and upcoming large cities in the vicinity of coral reefs, this issue remains of high importance.

Considering the temporal changes in biodiversity, it remains to be seen how species richness data of various areas can be compared in a biogeographical context (e.g., Edinger et al., 2000; Karlson et al., 2004). When species compositions of particular areas are compared, species records over longer periods are more comprehensive than only the most recent data. For the reefs off Jakarta, the record of 286 species of zooxanthellate scleractinians over the period 1920-2005 is higher than the 243 listed for 2005 only (Tables 2 and 3). This total of 286 species relates to numbers obtained from other reef systems in the proximity of large cities, such as the revised number of 255 scleractinians for Singaporean reefs, which also includes old and new records (Huang et al., 2009). A total of 262 recorded zooxanthellate scleractinians has been listed for the reefs off Makassar. This might be lower than expected because of an underrepresentation of the Acroporidae here (Best et al., 1989). When reef systems of Singapore, Jakarta, and Makassar are compared, the first appears to be entirely under siltation stress while the latter is situated in the Makassar Strait, where interoceanic currents pass and distinct onshore-offshore environmental gradients can be discerned (Hoeksema and Moka, 1989; Hoeksema, 2007). Regarding sponges, species richness data of reef systems near large cities follow a raising trend related to decreasing disturbance: 82 species at Singapore (de Voogd and Cleary, 2009), 118 at Jakarta (de Voogd and Cleary, 2008), and 151 at Makassar (de Voogd et al., 2006). However, this trend might be a biogeographic effect, reflecting a decreasing distance to the Coral Triangle (Hoeksema, 2007). Therefore it is important that in the present study a distinction is made between Jakarta Bay and the more offshore Thousand Islands, showing a clear species richness zonation between the two areas. Like in a similar case, involving molluscs (van der Meij et al., 2009), species loss in Jakarta Bay can therefore be attributed to the proximity of Jakarta and siltation from nearby river outlets.

When areas are compared for species richness, the available survey time and area may be limiting factors in obtaining reliable data for species-rich taxa. The use of species richness estimators may show whether monitoring time and habitat heterogeneity are sufficient. Alternatively, reliable data can be obtained in relatively short periods when smaller model taxa are compared, such as the mushroom coral family Fungiidae, with 15 species recorded in 2006 and 19 in 1860s-2006 at Singapore (Hoeksema and Koh, 2009), 28 at Jakarta in the present study, and 37 at Makassar (Hoeksema, 1989, 2007). The availability of historical collections and long-term data may be complimentary to recent surveys, as demonstrated by the present study and other recent ones (Hoeksema and Koh, 2009; Huang et al., 2009; van der Meij et al., 2009). These studies show that variation in time range and sampling effort can be underestimated factors in comparing species richness patterns at local, regional, and global scale. However, it should also be noted that in small areas, natural disturbances may play a more visible role in the dynamics of coral assemblages than in larger ones (Karlson and Cornell, 2002).

\section{Acknowledgements}

This publication is a result of the Kepulauan Seribu Expedition, September 2005, organized by the Research Centre for Oceanography, Indonesian Institute of Sciences (PPO-LIPI, Jakarta) and Naturalis. LIPI issued the research permits. The research was partly funded through the project 'Climate change and Indonesian coral reef biotas' within the research theme 'Biodiversity in relation to Global Change' of the Netherlands Organization for Scientific Research (ALW-NWO Grant 85200050). The digitalization of the coral collections of NCB Naturalis was conducted under the NWO-Groot project 'Building the databases of life'. The first author received a 2007 Mia J. Tegner Memorial Research Grant in Marine Environmental History and Historical Marine Ecology from the Marine Conservation Biology Institute, to elaborate and complete the present study. The A.M. Buitendijkfonds (Naturalis) and Leiden University funds funded her participation in the expedition. Ms. Aukje Coers, Mr. Bryan Meulens and Ms. Paola Rachello-Dolmen assisted with coral identifications.

\section{References}

Attahiyyat, C., 2000. Pulau Onrust. Dinas Museum dan Pemugaran Propinsi DKI Jakarta, pp. 1-65.

Bak, R.P.M., Nieuwland, G., 1995. Long-term change in coral communities along depth gradients over leeward reefs in the Netherlands Antilles. Bulletin of Marine Science 56, 609-619.

van Benthem-Jutting, W.S.S., Westenberg, J., ms. Mollusca of the Bay of Batavia (Java), and their importance for a biological valuation of the sea.

Best, M.B., Hoeksema, B.W., Moka, W., Moll, H., Suharsono, Sutarna, I.N., 1989. Recent scleractinian coral species collected during the Snellius-II Expedition. Netherlands Journal of Sea Research 23, 107-115.

Boschma, H., 1936. Sur la croissance de quelques coraux des récifs de l'ile d'Edam (Baie de Batavia). Mélanges Paul Pelseneer. Mémoires du Musée Royal d'Histoire Naturelle de Belgique Ser. 2 (3), 101-114.

Brown, B.E. (ed.), 1986. Human-induced damage to coral reefs. Results of a regional UNESCO (COMAR) workshop with advanced training, Diponegoro University, Jepara, and National Institute of Oceanology, Jakarta, Indonesia, May 1985 UNESCO Reports in Marine Science 40, 112-125.

Brown, B.E., Howard, L.S., 1985. Assessing the effects of "stress" on coral reefs Advances in Marine Biology 22, 1-63.

Brown, B.E., Suharsono, 1990. Damage and recovery of coral reefs affected by El Niño related seawater warming in the Thousand Islands, Indonesia. Coral reefs 8, 163-170.

Brown, B.E., Clarke, K.R., Warwick, R.M., 2002. Serial patterns of biodiversity change in corals across shallow reef flats in Ko Phuket, Thailand, due to the effects of local (sedimentation) and regional (climatic) perturbations. Marine Biology 141 21-29.

Bryant, D., Burke, L., McManus, J., Spalding, M., 1998. Reefs at risk. World Resources Institute, Washington, D.C. pp. 1-60.

Burke, L., Selig, E., Spalding, M., 2002. Reefs at risk in Southeast Asia. World Resources Institute, Washington, D.C. pp. 1-72.

Cairns, S.D., Hoeksema, B.W., van der Land, J., 1999. Appendix, list of extant stony corals. Atoll Research Bulletin 459, 13-46.

Chadwick-Furman, N.E., 1996. Reef coral diversity and global change. Global Change Biology 2, 559-568 
Cleary, D.F.R., Suharsono, Hoeksema, B.W., 2006. Coral diversity across a disturbance gradient in the Pulau Seribu reef complex off Jakarta, Indonesia. Biodiversity and Conservation 15, 3653-3674.

Cleary, D.F.R., DeVantier, L., Giyanto, Vail, L., Manto, P., de Voogd, N.J., RachelloDolmen, P.G., Tuti, Y., Budiyanto, A., Wolstenholme, J., Hoeksema, B.W. Suharsono, 2008. Relating variation in species composition to environmental variables, a multi-taxon study in an Indonesian coral reef complex. Aquatic Sciences 70, 419-431.

Connell, J.H., 1997. Disturbance and recovery of coral assemblages. Coral Reefs 16, $101-113$

Delsman, H.C., 1937. Marine biological investigations made by foreign visitors in the laboratory for marine investigation at Batavia until the year 1934. Extrait des Annales du Jardin Botanique de Buitenzorg 45, 121-128.

DeVantier, L., Suharsono, Budiyanto, A., Tuti., Y., Imanto, P., Ledesma, R., 1998 Status of coral communities of Pulau Seribu (Indonesia). In: Soemodihardjo, S. (Ed.), Contending with Global Change 10. Proceedings, coral reef evaluation workshop, Pulau Seribu, Jakarta, Indonesia, 1995. UNESCO, Jakarta, pp. 1-24.

Djohani, R.H., 1994. Patterns of spatial distribution, diversity, and cover of corals in Pulau Seribu National Park, implications for the design of coral sanctuaries. Proceedings IOC-WESTPAC 3rd International Science Symposium Bali, Indonesia, 265-279.

Edinger, E.N., Risk, M.J., 2000. Reef classification by coral morphology predicts coral reef conservation value. Biological Conservation 92, 1-13.

Edinger, E.N., Jompa, J., Limmon, G.V., Widjatmoko, W., Risk, M.J., 1998. Reef degradation and coral biodiversity in Indonesia, effects of land-based pollution, destructive fishing practices and changes over time. Marine Pollution Bulletin 36, 617-630.

Edinger, E.N., Kolasa, J., Risk, M.J., 2000. Biogeographic variation in coral species diversity on coral reefs in three regions of Indonesia. Diversity and Distributions 6, 113-127.

Fabricius, K.E., 2005. Effects of terrestrial runoff on the ecology of corals and coral reefs, review, and synthesis. Marine Pollution Bulletin 50 125-146.

Hardenberg, J.D.F., 1939. De koraaleilanden in de Baai van Batavia, Elfde verslag (1936-1938) van de Nederlandsch-Indische Vereeniging tot Natuurbescherming, pp. 234-242.

Hoeksema, B.W., 1989. Taxonomy, phylogeny, and biogeography of mushroom corals (Scleractinia, Fungiidae). Zoologische Verhandelingen Leiden 254, 1 295

Hoeksema, B.W., 1991. Control of bleaching in mushroom coral populations (Scleractinia, Fungiidae) in the Java Sea: stress tolerance and interference by life history strategy. Marine Ecology Progress Series 74, 225-237.

Hoeksema, B.W., 2004. Biodiversity and the natural resource management of coral reefs in Southeast Asia. In: Visser, L.E. (Ed.), Challenging Coasts, Transdisciplinary Excursions into Integrated Coastal Zone Development. Amsterdam University Press, Amsterdam, pp. 49-71.

Hoeksema, B.W., 2007. Delineation of the Indo-Malayan centre of maximum marine biodiversity, the Coral Triangle. In: Renema, W. (Ed.), Biogeography, Time and Place: Distributions, Barriers, and Islands. Springer, Dordrecht, pp. 117-178.

Hoeksema, B.W., Koh, E.G.L., 2009. Depauperation of the mushroom coral fauna (Fungiidae) of Singapore (1860s-2006) in changing reef conditions. Raffles Bulletin of Zoology Suppl. 22, 91-101.

Hoeksema, B.W., Moka, W., 1989. Species assemblages and ecomorph variation of mushroom corals (Scleractinia: Fungiidae) related to reef habitats in the Flores Sea. Netherlands Journal of Sea Research 23, 149-160.

Hooze, J.A., 1877. Artesische putboring op het eiland Onrust bij Batavia. In: Gemengde, geologische, technische en andere mededeelingen. Jaarboek van het Mijnwezen in Nederlandsch Oost-Indië 6, 190-194.

Huang, D.W., Yun, K.P.P., Chou, L.M., Todd, P.A., 2009. An inventory of zooxanthellate scleractinian corals in Singapore, including 33 new records. Raffles Bulletin of Zoology Suppl. 22, 69-80.

Hughes, T.P., Connell, J.H., 1999. Multiple stressors on coral reefs, a long-term perspective. Limnology and Oceanography 44, 932-940.

Hughes, T.P., Baird, A.H., Bellwood, D.R., Card, M., Connolly, S.R., Folke, C., Grosberg, R., Hoegh-Guldberg, O., Jackson, J.B.C., Kleypas, J., Lough, J.M., Marshall, P., Nyström, M., Palumbi, S.R., Pandolfi, J.M., Rosen, B., Roughgarden, J., 2003. Climate change, human impacts, and the resilience of coral reefs. Science 301, 929-933.

Jackson, J.B.C., Kirby, M.X., Berger, W.H., Bjorndal, K.A., Botsford, L.W., Bourque, B.J., Bradbury, R.H., Cooke, R., Erlandson, J., Estes, J.A., Hughes, T.P., Kidwell, S., Lange, C.B., Lenihan, H.S., Pandolfi, J.M., Peterson, C.H., Steneck, R.S., Tegner, M.J., Warner, R.R., 2001. Historical overfishing and the recent collapse of coastal ecosystems. Science 293, 629-637.

Karlson, R.H., Cornell, H.V., 2002. Species richness of coral assemblages: detecting regional influences at local spatial scales. Ecology 83, 452-463.

Karlson, R.H., Cornell, H.V., Hughes, T.P., 2004. Coral communities are regionally enriched along an oceanic biodiversity gradient. Nature $429,867-$ 870.

Marshall, P.A., Baird, A.H., 2000. Bleaching of corals on the Great Barrier Reef, differential susceptibilities among taxa. Coral Reefs 19, 155-163.

McCulloch, M., Fallon, S., Wyndham, R., Hendy, E., Lough, J., Barnes, D., 2003. Coral record of increased sediment flux to the inner Great Barrier Reef since European settlement. Nature 421, 727-730. van der Meij, S.E.T., Moolenbeek, R.G., Hoeksema, B.W., 2009. Decline of the Jakarta Bay molluscan fauna linked to human impact. Marine Pollution Bulletin 59, 101-107.

van der Meij, S.E.T., Visser, R.R., in press. The Acropora humilis group (Scleractinia) of the Snellius expedition (1929-30).

Moll, H., Suharsono, 1986. Distribution, diversity and abundance of reef corals in Jakarta Bay and Kepulauan Seribu. In: Brown, B.E. (ed.), Human-induced damage to coral reefs. Results of a regional UNESCO (COMAR) workshop with advanced training, Diponegoro University, Jepara, and National Institute of Oceanology, Jakarta, Indonesia, May 1985. UNESCO Reports in Marine Science 40, 112-125.

Munro, C., 2005. Diving systems. In: Eleftheriou, A., McIntyre, A. (Eds.), Methods for the study of marine benthos, third edition. Blackwell Science, Oxford, pp. 112159 .

Ongkosongo, O.S.R., 1989. Background information for the field trip to the Seribu Reefs. Indonesia - short course on carbonate sedimentology and stratigraphy, Indonesian Petroleum Association, pp. 1-270.

Razak, T.B., Hoeksema, B.W., 2003. The hydrocoral genus Millepora (Hydrozoa, Capitata, Milleporidae) in Indonesia. Zoologische Verhandelingen Leiden 345, 313-336.

Rees, J.G., Setiapermana, D., Sharp, V.A., Weeks, J.M., Williams, T.M., 1999. Evaluation of the impacts of land-based contaminants on the benthic faunas of Jakarta Bay, Indonesia. Oceanologica Acta 22, 627-640.

Risk, M.J., 1999. Paradise lost, how marine science failed the world's coral reefs. Marine and Freshwater Research 50, 831-837.

Roberts, C.M., 1993. Coral reefs, health, hazards, and history. Trends in Ecology and Evolution 8, 425-427.

Rogers, C.S., 1990. Responses of coral reefs and reef organisms to sedimentation. Marine Ecology Progress Series 62, 185-202.

Scheer, G., 1990. Die von E.J.C. Esper 1788-1809 beschriebenen Anthozoa (Cnidaria). Teil IV-V. SenckenbergianaBiologica 71, 369-429 (published 1991).

Schmitt, E.F., Sluka, R.D., Sullivan-Sealey, K.M., 2002. Evaluating the use of roving diver and transect surveys to assess the coral reef fish assemblage off southeastern Hispaniola. Coral Reefs 21, 216-223.

Sluiter, C.P., 1888. Die Evertebraten aus der Sammlung des königlichen naturwissenschaftlichen Vereins in Niederländisch Indien in Batavia, zugleich eine Skizze der Fauna des Java-Meeres, mit Beschreibung der neuen Arten. Natuurkundig Tijdschrift Nederlandsch Indië 47, 181-220.

Sluiter, C.P., 1890. Einiges über die Entstehung der Korallen in der Javasee un Branntweinsbai und über neue Korallenbildung bei Krakatau. Natuurkundig Tijdschrift Nederlandsch Indië 49, 360-380.

Stoddart, D.R., 1986. Umbgrove's Islands Revisited. In: Brown, B.E., (ed.), Humaninduced damage to coral reefs. Results of a regional UNESCO (COMAR) workshop with advanced training, Diponegoro University, Jepara, and National Institute of Oceanology, Jakarta, Indonesia, May 1985. UNESCO Reports in Marine Science 40, 112-125.

Sunier, A.L.J., 1923. The laboratory for marine investigations at Batavia, a new tropical marine biological station. Treubia 3, 127-148.

Sutamihardja, R.T.M., 1985. Water quality in the coastal area: the Jakarta Bay experience. In: Katō, I., Kumamoto, N., Matthews, W.H., Suhaimi, A. (Eds.), Environmental protection and coastal zone management in Asia and the Pacific. University of Tokyo Press, pp. 37-48.

Tomascik, T., Suharsono, Mah, A.J., 1993. Case histories: a historical perspective of the natural and anthropogenic impacts in the Indonesian archipelago with a focus on Kepulauan Seribu, Java Sea. In: Ginsburg, R. (Ed.), Proceedings of the colloquium on global aspects of coral reefs, Health, hazards and history. University of Miami, Rosenstiel School of Marine and Atmospheric Science, pp. 304-310.

Tomascik, T., Mah, A.J., Nontji, A., Moosa, M.H., 1997. The ecology of the Indonesian seas. Part two. Periplus, Singapore, pp. 1-745.

Umbgrove, J.H.F., 1928a. De Koraalriffen in de Baai van Batavia. Wetenschappelijke Mededeelingen 7 Dienst van den Mijnbouw in Nederlandsch-Indië.

Umbgrove, J.H.F., 1928. De invloed van den wind op de vorming der koraalriffen in de Baai van Batavia. De Tropische Natuur Jaargang 17, 123-130.

Umbgrove, J.H.F., 1929. De Koraalriffen der Duizend-Eilanden (Java Zee). Wetenschappelijke Mededeelingen 12. Dienst van den Mijnbouw in Nederlandsch, Indië.

Umbgrove, J.H.F., 1939. Madreporaria from the Bay of Batavia. Zoologische Mededelingen Leiden 22, 1-64.

Umbgrove, J.H.F., Verwey, J., 1929. The coral reefs in the Bay of Batavia. Fourth Pacific Science Congress Java 1929.

Uneputty, P.A., Evans, S.M., 1997. Accumulation of beach litter on islands of the Pulau Seribu Archipelago, Indonesia. Marine Pollution Bulletin 34, 652-655.

Veron, J.E.N., 2000. Corals of the World. Vol. 1-3. Australian Institute of Marine Science and CRR, Queensland, Australia.

Veron, J.E.N., Pichon, M., 1976. Scleractinia of Eastern Australia - Part I. Families Thamnasteriidae, Astrocoeniidea, Pocilloporidae. Australian Institute of Marine Science Monograph Series 1, 1-86.

Veron, J.E.N., Pichon, M., 1980. Scleractinia of Eastern Australia - Part III. Family Agariciidae, Siderastreidae, Fungiidae, Oculinidae, Merulinidae, Mussidae, Pectinidae, Caryophyllidae, Dendrophylliidae. Australian Institute of Marine Science Monograph Series 4, 1-459.

Veron, J.E.N., Pichon, M., 1982. Scleractinia of Eastern Australia - Part IV Family Poritidae. Australian Institute of Marine Science Monograph Series 5, 1-159. 
Veron, J.E.N., Wallace, C.C., 1984. Scleractinia of Eastern Australia - Part V. Family Acroporidae. Australian Institute of Marine Science Monograph Series 6, 1485.

Veron, J.E.N., Pichon, M., Wijsman-Best, M., 1977. Scleractinia of Eastern Australia Part II. Families Faviidae, Trachyphylliidae. Australian Institute of Marine Science Monograph series 3, 1-233.

Verstappen, H.T., 1953. Djakarta Bay - a geomorphological study on shoreline development. PhD-thesis, University of Utrecht, pp. 1-101.

Verstappen, H.T., 1988. Old and new observations on coastal changes of Jakarta Bay, an example of trends in urban stress on coastal environments. Journal of Coastal Research 4, 573-587.

Verwey, J., 1931. Coral reef studies II, III. Treubia 13, 169-198.

de Voogd, N.J., Cleary, D.F.R., 2008. An analysis of sponge biodiversity and distribution at three taxonomic levels in the Thousand Islands/Jakarta Bay reef complex, West-Java, Indonesia. Marine Ecology 29, 205-215.

de Voogd, N.J., Cleary, D.F.R., 2009. Variation in sponge composition among Singapore reefs. Raffles Bulletin of Zoology Suppl. 22, 59-67.

de Voogd, N.J., Cleary, D.F.R., Hoeksema, B.W., Noor, A., Soest, R.W.M. van, 2006. Sponge beta diversity in the Spermonde Archipelago, Indonesia. Marine Ecology Progress Series 309, 131-142.

Wallace, C.C., 1999. Staghorn Corals of the World, A Revision of the Coral Genus Acropora. CSIRO, Collingwood, Australia.

Wallace, C.C., Wolstenholme, J., 1998. Revision of the coral genus Acropora (Scleractinia, Astrocoeniina, Acroporidae) in Indonesia. Zoological Journal of the Linnean Society 123, 199-384.

Warwick, R.M., Clarke, K.R., Suharsono, 1990. A statistical analysis of coral community responses to the 1982-83 El Niño in the Thousand Island, Indonesia. Coral Reefs 8, 171-179.
Wichmann, C.E.A., 1912. Over de zoogenaamde atollen van den Oost-Indischen Archipel. Koninklijke Akademie van Wetenschappen te Amsterdam 641, 654.

Wielgus, J., Glassom, D., Fishelson, L., 2003. Long-term persistence of low coral cover and abundance on a disturbed coral reef flat in the northern Red Sea. Journal of Experimental Marine Biology and Ecology 297, 31-41.

Wijsman-Best, M., 1972. Systematics and ecology of New Caledonian Faviinae (Coelenterata - Scleractinia). Bijdragen tot de Dierkunde 42, 1-90.

Wilkinson, C.R., 1993. Coral reefs of the world are facing widespread devastation, can we prevent this through sustainable management practices? Proceedings of the 7th international coral reef symposium. Guam 1, 11-21.

Wilkinson, C.R., 1996. Global change and coral reefs, impacts on reefs, economies, and human cultures. Global Change Biology 2, 547-558.

Willoughby, N.G., 1986. Man-made litter on the shores of the Thousand Island archipelago, Java. Marine Pollution Bulletin 17, 224-228.

Willoughby, N.G., Sangkoyo, H., Lakaseru, B.O., 1997. Beach litter: an increasing and changing problem for Indonesia. Marine Pollution Bulletin 34, 469-478.

Wilson, S.K., Graham, N.A.J., Pratchett, M.S., Jones, G.P., Polunin, N.V.C., 2006 Multiple disturbances and the global degradation of coral reefs, are reef fishes at risk or resilient? Global Change Biology 12, 2220-2234.

Zaneveld, J.S., Montagne, D.G., 1950. 'Boompjes-eiland' een koraaleiland in de Javazee. Extract from the periodical K.N.A.G. (Koninklijk Nederlands Aardrijkskundig Genootschap) 67, 715-745.

Zaneveld, J.S., Verstappen, H.T., 1952. A recent investigation about the geomorphology and the flora of some coral islands in the Bay of Djakarta. Journal for Scientific Research 3, 58-68. 